\title{
Zur Verankerung demografiebezogener Themen in Tarifverträgen und Betriebsvereinbarungen - Beobachtungen und Einschätzungen
}

P. Guggemos und R. Conrads

\section{Einleitung}

Nachstehender Aufsatz stellt zunächst anhand einiger Leitfragen wissenschaftliche Betrachtungsperspektiven auf das Thema der Verankerung und Regulierung demografiebezogener Themenfelder vor. In einem zweiten Teil wird erhellt, was aus Sicht der Wissenschaft und der Betriebe zum Demografiemanagement ${ }^{1}$ gehört. Damit sollen diagnostische wie umsetzungsorientierte Maßnahmen in den Blick genommen werden, die verbreitet auf einen inzwischen auch in seiner Dringlichkeit wahrgenommenen Handlungsbedarf reagieren und deshalb tätigkeits- und branchenübergreifend als bedeutsam angesehen werden. Diese sind unabhängig von Maßnahmen zu sehen, die auf tätigkeits- bzw. branchenspezifische Herausforderungen reagieren. Ein dritter Abschnitt zeigt Beispiele aus Tarifverträgen und Betriebsvereinbarungen, sowie letztlich nicht zustande gekommene Regulierungsansätze und resümiert den Forschungsstand. Im vierten Teil wird eine zusammenfassende Deutung zum Stand der Umsetzung betrieblicher Demografiepolitik über Tarifverträge und Betriebsvereinbarungen versucht, und abschließend werden einige tarifpolitische Perspektiven und Handlungsempfehlungen aufgezeigt.

\section{Leitfragen und Vorüberlegungen:}

1.1 Welche Themenfelder geraten unter dem Label „Demografie-bezogener Handlungsbedarf" ins Blickfeld, und welchen wird jeweils ein besonderer Handlungsbedarf zugemessen?

Der Fokus besteht hier in den identifizierten Herausforderungen und Aufgaben für die Politik, für Unternehmen und, noch etwas genauer, für Firmenleitungen, Personalverantwortliche und Mitarbeitervertretungen, sowie für Personen in unterschiedlichen Tätigkeits- und Lebensweltbedin- 
gungen. Der Zweck der wissenschaftlichen Auseinandersetzung ist hierbei, die jeweiligen Deutungsrahmen (,frames")2 herauszuarbeiten. Angenommen werden darf, dass in diese neben je eigenen Interessenlagen auch unterschiedliche Fachlichkeiten und Kenntnisse im Personal- und Demografiemanagementbereich, (betriebs-)sozialisatorische Erfahrungen, aktuell als bedeutsam eingestufter Handlungsbedarf, sowie eine Einschätzung von Erfolgswahrscheinlichkeiten, Aufwand und Ertrag einzelner Maßnahmen und Maßnahmenstränge mit eingehen.

\subsection{Welche Handlungsfelder erachten Tarifparteien nach ibren Erfahrungs- werten für weitestgehend branchenweit tarifpolitisch gestaltbar und für Firmen unterschiedlicher Größe, Fachkompetenz und ökonomischer Situation anwendbar?}

Hierbei besteht der wissenschaftliche Auftrag darin, einzelne beschlossene Tarifverträge beispielhaft mit einem branchendifferenzierenden und Unterschiede einfangenden Blick zu analysieren. Es sind ferner Veränderungen im Zeitverlauf im Sinne von Weiterentwicklungen, Paradigmenwandeln bis -wechseln und ggf. auch aufgegebene Ziele und Maßnahmen herauszuarbeiten, sowie Schubladenentwürfe und gescheiterte/nicht beschlossene Vorhaben ebenso mit zu betrachten, wie Einschätzungen beteiligter Akteure inklusive eines Zukunftsausblicks zumindest selektiv mit einzubeziehen. Angenommen werden darf hierbei, dass Tarifverträge Kompromisse darstellen zwischen in Teilbereichen divergenten Interessenlagen, und dass Themenfelder mehr Chancen auf Durchsetzung haben, die von mehreren Seiten als wichtig und zugleich mit vorhandenen Ressourcen (Personal, Know-how, Geld) als machbar erachtet werden. Umgekehrt dürften es all diejenigen Handlungsfelder schwer haben, die als personal-, wissensund/oder kostenintensiv eingeschätzt werden, und/oder deren Erfolgswahrscheinlichkeit und/oder Kosten-Nutzen-Relation als unsicher bis schlecht erscheint. Handlungsfelder, die jeweils nur oder zumindest ganz überwiegend einer anderen Interessengruppe wichtig erscheinen, einem selbst aber nicht, dürften dann eine Chance auf Durchsetzung haben, wenn sie Teil einer Verhandlungsmasse über Paketlösungen sind. 


\subsection{Bei welchen Themenfeldern besteht eine hohe Wahrscheinlichkeit, dass die anvisierten Maßnahmen auch in hinreichender Qualität realisiert werden und den erwarteten Nutzen bringen dürten?}

Diese Frage zielt zum einen auf das gegenseitige Vertrauen der Verhandlungspartner*innen in den jeweiligen Umsetzungswillen, aber auch auf eine Kompetenzeinschätzung der Umsetzungsakteur*innen ab. Und umgekehrt ist zu fragen, wo mit welchen Umsetzungswiderständen zu rechnen ist, und wie diese konstruktiv bearbeitet werden könnten. Es ist zu erwarten, dass einerseits betriebsindividuell mehr möglich erscheint als auf Tarifvertragsebene, und andererseits, dass nur ein Teil der State-of-the-artMaßnahmen im eigenen Betrieb als umsetzbar eingestuft und fixiert wird. Ressourcenschwächere Unternehmen orientieren sich fallweise an einzelnen tarifvertraglichen Regelungen, haben aber möglicherweise selbst den Tarifvertrag nicht unterzeichnet oder sich einer anderen Tarifgemeinschaft mit abgeschwächten Standards angeschlossen ${ }^{3}$. Aufgabe der Wissenschaft ist es schließlich, anhand der Befragung betrieblicher Akteure derartige Regelungen und deren Umsetzung in Erfahrung zu bringen. Tarifpolitiker*innen sowohl von Arbeitgeber- wie Arbeitnehmerseite haben derartige Vorhaben und Unterschiede zwischen Vorreitern und Nachzüglern/ Abweichenden mit im Blick, und entwickeln entsprechende Ausnahmeklauseln. Zugleich werden Regelungen möglichst ausnahmslos für alle definiert und es werden Auffangregeln entworfen, die dann Gültigkeit erlangen, wenn vorgesehene Angebote bzw. Gestaltungsmöglichkeiten (beispielsweise zur Nutzung eines Demografiefonds) nicht realisiert werden sollten. Unter den Aspekten der Legitimation, der Rücksicht auf kleinere und ressourcenärmere Betriebe und des Schutzes vor Überforderung müssen tarifpolitische Akteure Komplexität einhegen, mutmaßliche Umsetzungshürden antizipieren und Unterstützungsmöglichkeiten eruieren. Alle Fragen der Umsetzung im betrieblichen Alltag inklusive der Probleme sind zu berücksichtigen, die das Herstellen der individuellen Einzelfallgerechtigkeit bei der Umsetzung aufwirft, wenn beispielsweise jeweils komplexe Anspruchsvoraussetzungen geprüft, entschieden und gerechtfertigt werden müssen. Zu beachten ist hier insofern die Schnittstelle vom Altersund Alternsmanagement zum Diversity Management, als neben „Maßnahmen für Ältere" weitere einzelpersonen- und gruppenbezogene Bedürfnisse treten. 


\subsection{Welche Handlungsfelder und welche Maßnahmen wurden mit welchem} Erfolg bearbeitet, und welche haben sich in der Umsetzung als suboptimal, bürdenreich und sperrig erwiesen und zeigen Nachsteuerungsbedarf auf?

Aus wissenschaftlicher Sicht wären hier das Zusammenspiel der Einzelmaßnahmen und deren Integration zu einem System ebenso als wünschenswert zu betrachten wie Lernanregungen und Vorbilder, sowie eine Prozessentwicklung hin zu einem sog. „Lernenden System“ mit einzelfallunabhängigen Regelungen, Routinen und Kompetenzen. Angesichts einer je nach betriebsindividuellen Besonderheiten wie unterschiedlicher Ressourcenausstattung zu erwartenden Streubreite hinsichtlich der Umsetzungserfolge in den jeweiligen Handlungsfeldern wie im Demografiemanagement insgesamt suchen die - selbst sich wiederum in heterogene Teildisziplinen wie Arbeitswissenschaft, Arbeitsmedizin, Industriesoziologie usw. differenzierende und teils arbeitgeber- und teils arbeitnehmernähere - Wissenschaft und Berater*innenlandschaft üblicherweise nach umsetzungsbegünstigenden und -hemmenden Faktoren. Daraus resultieren Bestrebungen, generalisierbare Umsetzungsempfehlungen, Handlungskonzepte und Stufenmodelle, sowie Unterstützungsangebote durch Dritte herauszuarbeiten. Dies soll anhand guter Praxisbeispiele und Befragungen beteiligter Entscheidungs- wie operativer Akteure sowie Maßnahmenbetroffener erfolgen.

\subsection{Welche Diskrepanzen bestehen zwischen den Fachdiskursen in der Wissenschaft und den einschlägigen Fachinstitutionen (wie BAuA, Sozialversicherungen etc.) einerseits, und andererseits der betrieblichen Realität, und wie erklären sie sich?}

Dies zu hinterfragen hat sich für das Verständnis betrieblichen Handelns als besonders hilfreich erwiesen, insofern es zutage gefördert hat, dass dortiges Agieren eben im Regelfall gerade nicht von der Theorie und dem Buchwissen zur Praxis verläuft, sondern sich häufig aus konkretem Handlungsbedarf vor Ort und der Bearbeitung anstehender Einzelfälle ergibt, woraus entgegen dem Idealfall aber nicht zwangsläufig systemische Lernprozesse entstehen. In der Realität der Tarifpolitik würde man hier von „Prozesstarifverträgen“ sprechen, d.h. in aufeinander bezogenen Stufen entlang einer längeren, die Gültigkeitsdauer einzelner Tarifverträge deutlich überschreitenden Zeitschiene sollen Handlungssysteme und Kompetenzen iterativ aufgebaut werden. Ein solches Vorgehen ist auch als ein Signal dafür zu verstehen, dass Akteuren, die eher eine Minimalumsetzung 
anvisieren, von vornherein klar sein dürfte, dass sie diese ersten festgeschriebenen Stufen im Kontext eines längeren Weges sehen und als Impulse zu weiterreichendem Handeln (mit hierzu nötigen Fachkompetenzen) deuten sollten. Im nachstehenden empirisch begründeten Entwicklungsmodell (Abbildung 1) ist zu erkennen, auf welchen unterschiedlichen IstStufen sich einzelne Betriebe aktuell befinden, und wohin sie sich entwickeln könnten.

\section{Abbildung 1: Modell Reifegrad des Demografiemanagements im Betrieb}

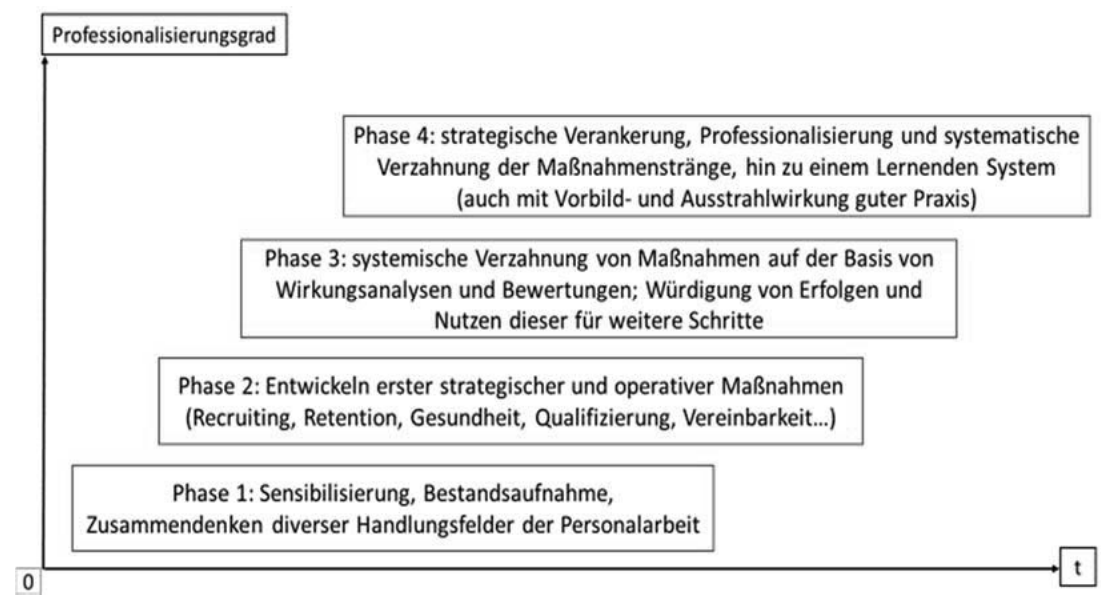

Quelle: eigene Darstellung 2020

\subsection{Welche Heterogenität an Aufgabenstellungen und Prämissen ergibt sich aus Tätigkeits- und Branchenunterschieden?}

Die erkennbaren Differenzen erklären mit, weshalb es einerseits der Konkretisierung bestimmter Regulierungsbereiche in Betriebsvereinbarungen bedarf, und weshalb andererseits manche Regelungen auf Konzernebene nicht für alle Unternehmensteile bzw. auch Personengruppen übernommen werden ${ }^{4}$. Anhand der Abbildung 1 oben lässt sich feststellen, wo der jeweilige Betrieb oder Betriebsteil momentan steht, und wohin er sich im nächsten Schritt mutmaßlich entwickeln dürfte. Die Schritte 1 bis 4 sind der übliche Entwicklungsweg, es ist aber nicht ausgeschlossen, dass sich ein Unternehmen ambitionierte Ziele setzt und gleich Phase drei oder vier anvisiert. Das Modell soll zugleich dafür sensibilisieren, dass es für einen noch kaum geregelten Bereich mit erst minimalen Personalressourcen und 
Qualitätsstandards „Guter Arbeit“ schon ein enormer und nur mit großen Anstrengungen zu erreichender Fortschritt sein könnte, auf Stufe 1 oder 2 $\mathrm{zu}$ kommen, und es möglicherweise leichter für ein Unternehmen mit langjähriger Erfahrung in anspruchsvoller Personalarbeit ist, seinen höheren Stand (z.B. Phase 3) zu halten oder noch auszubauen.

Eingebettet sind obige Fragen in einen Kontext, der in systemvergleichender Perspektive deutlich macht, dass in dem Moment, wo korporatistische Aushandlungssysteme zwischen dem Staat und den Verbandsakteuren des Arbeitsmarktes schwächer werden oder gar nicht (mehr) existieren, die Aushandlungsebenen der Tarifverträge bzw. der Betriebsvereinbarungen an Bedeutung für eine sozialpolitische Absicherung zunehmen ${ }^{5}$. Umgekehrt wäre in einem Land wie Österreich als einem prototypischen neokorporatistischen Land das tripartistische Verhandlungssystem zwischen dem Staat und den sog. „Sozialpartnern“ auf der jeweiligen föderativen Ebene die erste Adresse, über die die jeweilige sozialpolitische Rahmensetzung erfolgen müsste.

In Deutschland nahm die Bedeutung der betrieblichen Altersvorsorge (neben der privaten) mit der prozentualen Absenkung der Rentenrelation zum vorherigen Erwerbseinkommen $\mathrm{zu}^{6}$.

\section{Demografiemanagement aus wissenschaftlicher und betrieblicher Warte}

In diesem Abschnitt wird der Frage nachgegangen, was wichtige demografiebezogene Erkenntnisse für Firmen sind, und welche Handlungsfelder und Instrumente diesbezüglich zum Einsatz kommen sollen. Die Erkenntnisse sind dabei zweigeteilt, d. h. es geht einmal um die Befunde des Fachdiskurses, also darum, was von Expert*innenseite für relevant erachtet wird, um Firmen demografiefest zu machen, und zum anderen, wo sich auf Basis konkreter betriebs- und abteilungsbezogener Daten und deren Deutung inklusive einer strategischen Vorschau - welcher konkrete Handlungsbedarf zeigt, und wie dieser angegangen werden soll. Wenn der wissenschaftliche Diskurs auf der betrieblichen Ebene Wirkmächtigkeit entfalten soll, muss er folglich mit den konkreten betrieblichen Zahlen, Rahmenbedingungen und Handlungsmöglichkeiten eng verzahnt werden. Da die Entscheidung über die Maßnahmen den (tarif-)betrieblichen Akteuren und eben gerade nicht der Wissenschaft obliegt, kommt der Wissenschaft eine beratende und Einfluss nehmende Funktion, aber keine Entscheidungs- und Festlegungskompetenz zu. In den vergangenen 20 Jahren konnte die Wissenschaft 
1. generalisierte Modelle mit klaren Handlungsfeldern entwickeln, die in ihrem Zusammenspiel und in ihrer Zusammenschau ein „Demografiemanagement" umreißen. Diese Modelle sind einerseits abstrakt genug, um ubiquitär Verwendung finden zu können, und andererseits wiederum konkret genug, um zumindest die groben Handlungsfelder vorzugeben und deren Zusammenhang aufzuzeigen. Wichtigstes diesbezügliches Modell ist das „Haus der Arbeitsfähigkeit“ von Juhani Ilmarinen und Jürgen Tempel (2013). Weiterhin wichtig sind das Konzept der differenziellen Arbeitsgestaltung (Ulich 2016) oder das auf dem Arbeitsfähigkeitsansatz aufbauende AGE-Management-Konzept (Hafesbrink \& Krause 2017).

2. in Kooperation mit Institutionen wie der BAuA, der Initiative Neue Qualität der Arbeit (INQA), PsyGA, dem Demografieberaternetzwerk ddn, zahlreichen kleineren und größeren Forschungs- und Beratungsinstituten Instrumente und Handlungskonzepte bereitstellen, deren Anwendung teils umstritten (Arbeitsbewältigungsindex/Work Ability Index), teils tarifvertraglich verordnet (Altersstrukturanalysen mit Vorschau) und teils sogar gesetzlich vorgeschrieben ist (betriebliches Eingliederungsmanagement). Angestrebt ist hierbei von Wissenschaftsseite die Ausweitung sowie auch die zielführende Anwendung der Tools auf möglichst alle Branchen und Personenkreise bis hin zu Langzeitarbeitslosen.

3. Akteure aus Politik und Verwaltungen dazu motivieren, Mittel für Forschungen und Umsetzungen entsprechender Erkenntnisse (z.B. als regionale und/oder betriebsbezogene Demografieberatung) bereitzustellen, wobei manchmal aber lange nicht immer der - oftmals angemahnte - Übergang von einer temporären Projektförderung zu einer verstetigten Regelförderung gelang.

4. spezifische Herausforderungen in Regelungsbereichen, Branchen und Tätigkeitsfeldern untersuchen, und auf dieser Basis Handlungsbedarf aufzeigen, Regulierungsvorschläge machen und Transfermöglichkeiten in andere Branchen aufzeigen.

5. Arbeitgeber- und Arbeitnehmerakteure bei der Formulierung tarifpolitischer Anliegen und der Verankerung in Tarifverträgen und Betriebsvereinbarungen unterstützen, wobei diese Kooperation von Wissenschafts- wie von Betriebsseite teils als Holschuld und teils als Bringschuld gesehen und entsprechend nachfrage- oder angebotsseitig eingeleitet wurde.

Aus betrieblicher Sicht zeigen sich unter der Rubrik „Demografiemanagement", die für viele Betriebe bis heute keine oder zumindest keine klare 
Kategorie ist, weshalb sie lieber bei den traditionellen Aufgaben des Personalmanagements bleiben und diese auch nicht speziell unter dem Altersfokus sehen ${ }^{7}$, insbesondere folgende Aufgaben:

1. sich einen systematischen Überblick darüber zu verschaffen, wer wann vermutlich in Rente gehen wird, und was das für den Betrieb und seine Altersstruktur bedeutet. Damit einher gehen Überlegungen, welche Aufgaben auf wen übertragen werden können und sollen, was hinsichtlich der Anforderungsprofile für Nachfolger*innen beibehalten und was modifiziert werden soll, und woher die Nachfolger*innen rekrutiert werden könnten. Bei Altersstrukturanalysen ist eine Differenzierung nach Arbeitsbereichen und Funktionen nötig, da es beispielsweise sein könnte, dass alle Meister*innen eines kleinen Betriebes schon knapp 60 sind, ohne dass die ganzen Teams überaltert sein müssten.

2. das Gewinnen von Nachwuchskräften, teils durch eigene Ausbildung und teils durch Rekrutieren (vor allem akademisch gebildeter Personen) am Markt, sowie das Halten von Beschäftigten.

3. Überlegen, was mit leistungsgewandelten Personen geschehen könnte $^{8}$, angesichts verstellter Optionen der Frühverrentung. Zu denken ist hier an Möglichkeiten der Arbeitsgestaltung und der Arbeitsteilung in Teams, der Verbreiterung der Einsatzfähigkeit der Beschäftigten durch Weiterbildungen und Job-Rotation (auch z.B. zwischen Komponenten- oder Vorfertigung im Betrieb und Arbeiten beim Kunden), Job Enrichment und Job Enlargement (Arbeiten in selbstorganisierten Teams mit ganzheitlicheren Arbeitsgängen, gegenseitiger Vertretungsmöglichkeit, und immer wieder neu vereinbarter Arbeitsteilung), der Schaffung weniger beanspruchender aber kompetenzadäquater Formate (ohne Stigmatisierung und Diskriminierung derjenigen, die in diesen Formaten arbeiten), sowie des Zusammenspiels von Erfahrungswissen und neuen Ausbildungsinhalten und Fachkompetenzen Jüngerer. ${ }^{9}$

4. Vor allem bei größeren Firmen stellt sich die Frage, was getan werden könnte, um präventiv die Beschäftigungsfähigkeit der Belegschaft zu erhalten. Hierbei zeigt sich, unterhalb dieses tarifpartnerübergreifenden Konsenses eine unterschiedliche Fokussierung, die mit gewisser Vorsicht so zu deuten ist, dass in manchen Branchen wie Metall auch auf Gewerkschaftsseite sowie überwiegend auf Arbeitgeberseite ein starkes Augenmerk auf Weiterbildung gerichtet ist, während das Thema der Belastungsreduktion und des altersgerechten Arbeitens vielfach immer noch vor allem ein Arbeitnehmeranliegen ist und sich Arbeit- 
geber*innen nicht unbedingt dafür zuständig sehen, und eher fürchten, hier ob schwammiger Grenzziehungen ein Einfallstor für vielerlei Wünsche und unkontrollierbare Folgekosten zu bekommen. ${ }^{10} \mathrm{Im}$ Bereich des Gesundheitsmanagements bzw. betrieblicher Gesundheitsförderung hebt die Arbeitgeberseite tendenziell stärker auf Verhaltensprävention und die Arbeitnehmerseite eher auf verhältnispräventive Bemühungen ab. Belastungsbezogene Auseinandersetzungen können von Gewerkschaftsseite auch zum individuellen Empowerment und zum politischen Agenda Setting genutzt werden ${ }^{11}$.

5. Regelungen entwickeln zur Linderung, Kompensation und zu Tätigkeitswechseln zur Bewältigung berufstypischer Herausforderungen, zum Schutz vor Überforderung und zur Regeneration und insgesamt zum Erhalt der Arbeits- und Beschäftigungsfähigkeit. Beispiele hierfür sind u.a.

- körperlich anstrengendes Arbeiten (schweres Heben und Tragen bspw. beim Flughafen-Bodenpersonal, bei Dachdeckern und Pflasterern, diversen Pflegetätigkeiten gerade auch in der häuslichen Pflege; häufige Nachtschichten),

- Exposition gegenüber Hitze/Kälte/Feuchtigkeit/Witterungsbedingungen (Baustellenberufe, Zustellung, Arbeit in Kühllagern),

- psychisch stark belastende Tätigkeiten (z.B. Beschwerdemanagement, Erziehungsberufe, Callcenter, Notärzte, Piloten, Pflegekräfte in Unterbesetzung, Tätigkeiten mit Anteilen an Emotionsarbeit $^{12}$ von Steward*ess bis Bedienung),

- olfaktorische Belastungen (Abfallverwertung, Schlachthof, Tierfutterproduktion, Tierkörperverwertung),

- permanente Freundlichkeitserwartungen (z.B. Flugbegleiter*innen, Supermarktkassierer*innen, Service-Kräfte in Hotel \& Gastro),

- Arbeiten in oft aversivem Kundenumfeld (Polizei, Ordnerdienste, Zugbegleiter*innen und Bahn-Infopoint-Beschäftigte, Arbeitsverwaltung).

6. Der Aufbau von Gesundheitsressourcen, der u.a. der Kompensation (oft unabänderlicher) o.g. tätigkeitsspezifischer Belastungen dient. Eine Abmilderung und ein Ausgleich ungünstiger Arbeitsbedingungen reduzieren den Krankenstand und die Fluktuationsquote. Trotz bekannt hoher Belastungen und Schwierigkeiten, hinreichend Personal zu rekrutieren, sind in einigen Branchen leider selbst kleine Verbesserungen oft nur um den Preis harter Auseinandersetzungen zwischen den Tarifparteien zu haben (Beispiel Krankenhauspersonal). Von Gewerkschaftsseite wird perspektivisch betont, dass es ihnen ein 
Anliegen ist, die Kolleg*innen nicht nur „gesund bis zur Rente“ zu erhalten, sondern sie auch noch gerne "gesund in der Rente" sehen zu wollen ${ }^{13}$.

7. das Management lebensweltlicher Herausforderungen, die die Verfügbarkeit für die Erwerbsarbeit beeinträchtigen, wie Kinderbetreuung (vor allem in Ferien und Randzeiten) und Pflegeaufgaben, was i.d.R. durch Arbeitszeitreduktion bzw. temporäre Freistellungen über individuelle Vereinbarungen gelöst wird. Eine Unterstützung der Care-Aufgaben selbst und eine generelle Verbesserung der Vereinbarkeitsarrangements wird nur von einer Minderheit der Betriebe ${ }^{14}$ als Firmenaufgabe gesehen - und wenn, dann eher von größeren und von Unternehmen mit hohem Frauenbeschäftigtenanteil. Punkt 7 zeigt die Notwendigkeit der Erweiterung vom „Altersmanagement“ zum „Lebensphasenmanagement" ${ }^{\text {" } 15}$, und weitet den Blick dafür, dass die genannten Care-Aufgaben in unterschiedlichem kalendarischem Alter eintreten können. Interessant ist diesbezüglich die Beobachtung, dass sich hier ein Feld für „Employer Branding“ zeigt. Dieser Vorteil bleibt allerdings nur solange erhalten, bis diesbezügliche Bemühungen überall zum Standard betrieblicher Leistungen zählen, und nicht mehr nur in Betriebsvereinbarungen, sondern in generellen Tarifverträgen ihren Niederschlag finden. Für Firmen mit besserem Vereinbarkeitsarrangement zeigt sich, dass dadurch familienbedingte Auszeiten signifikant reduziert werden können, da Beschäftigte früher aus Elternphasen zurückkehren ${ }^{16}$.

8. Bemühungen zum Erhalt und zur Stärkung des psychologischen Arbeitsvertrages. Hierzu zählen, neben betrieblichen Feiern und Ausflügen, die es meist auch in kleineren Betrieben gibt, Führungstrainings und Bemühungen zur Humanisierung der Arbeitswelt, in der Hoffnung im Gegenzug ebenfalls mehr Engagement und Einsatzflexibilität der Beschäftigten zu bekommen. Unterstützung bei der Stressbewältigung und bei der Linderung von Belastungsfolgen einseitiger Körperhaltungen dienen sowohl dem Commitment wie der Reduktion von Fehltagen, wobei festzustellen ist, dass zwischen gesundheitsorientierten Maßnahmen und Mitarbeiterzufriedenheit bzw. -gesundheit kein unikausaler, sondern eher ein vermittelter Zusammenhang besteht, bei dem sich die intendierte Botschaft der Maßnahmenverantwortlichen und die Wahrnehmung durch deren Adressaten durchaus unterscheiden können. Bei den Maßnahmen ist insbesondere wichtig, dass diese partizipativ konfiguriert und dadurch legitimiert wurden.

9. Kompensation ausgelaufener Vorruhestandsmöglichkeiten durch unternehmenseigene Ansparmodelle. Ein Teil des Lohns wird dabei in 
einen Fonds einbezahlt, aus dem für eine Übergangszeit eine Arbeitszeitreduktion und am Ende ein vorzeitiger Ruhestand finanziert werden können. (Beispiel: Post). Aus Arbeitnehmersicht ist dabei wichtig, dass die Zeit vor dem vorgezogenen Ruhestand nicht mit allzu großem Gehaltsverzicht einhergeht, und dass sich der vorgezogene Altersübertritt nicht zu rentenmindernd auswirkt. Wichtig scheint hier der Hinweis, dass zwar viele Ökonomen und Alter(n)swissenschaftler*innen das Ziel eines prolongierten Erwerbslebens für alternativlos halten, das Meinungsspektrum der Belegschaften hierzu jedoch breit streut: Wo die einen den vorgezogenen Ruhestand herbeisehnen (insbesondere in Umstrukturierungsphasen mit hohen Lernherausforderungen), wollen andere nochmal durchstarten und lieber länger als kürzer arbeiten ${ }^{17}$.

10. Absicherung nachlassender bis nicht mehr gegebener Arbeitsfähigkeit. Risikoabsicherungsmodelle arbeiten ebenfalls mit einem Fonds, der allerdings nur bei Bedarf angezapft werden kann, wodurch nur ein Teil der Belegschaft in dessen Genuss kommt, und zudem innerbetrieblich anhand definierter Kriterien entschieden werden muss, ob und wann die Anspruchsvoraussetzungen gegeben sind.

11. Schaffen von Entlastung für bestimmte Bedingungen (alter $(\mathrm{n})$ sgerechte Arbeitszeitregulierungen, z.B. bei Vollkonti-Schicht, Dauer-Nachtschicht etc.) und ab einem definierten kalendarischen Alter. Die Demografieverträge der Chemie haben hierfür wöchentliche Stundenreduktionen vorgesehen, die zu Freischichten zusammengefasst werden können. Dadurch entsteht Erleichterung im laufenden Arbeitsbetrieb, aber kein vorgezogener Ruhestand. Die Akzeptanz dieser Regelungen ist insgesamt auch bei Jüngeren gut, wenngleich es gelegentlich organisatorische Herausforderungen in den Betrieben gibt und die Freischichten etwas despektierlich als „Grufti-Schichten“ bezeichnet wer$\operatorname{den}^{18}$.

12. Management von Umstrukturierungen, technisch-organisatorische Anpassungen an neue Prozessroutinen und die Organisation von Schulungen sowie die Herstellung von Flexibilität und Umstellungsbereitschaft, v.a. im fortgeschrittenen Alter. Ein regelmäßiger Schulungsbedarf ergibt sich ferner aus rechtlichen Sicherheitsvorgaben und Zertifizierungsvorschriften (Beispiele Chemie, Auftragsfertigung).

13. mitunter die Durchführung von Orientierungsworkshops im Alter um die 50 bis 55 Jahre $^{19}$, um herauszufinden, was nötig wäre von Arbeitnehmer- wie Arbeitgeberseite, um die betreffenden Personen bis zur Rente gesund, qualifiziert und motiviert zu halten, und eine Balance zwischen privat-lebensweltlichen und dienstlichen Belangen herzustellen. Hier geht es u.a. darum, Entwicklungsmöglichkeiten und Lernge- 
legenheiten auch für Personen zu schaffen, die ihre angestrebte Karrierestufe längst erreicht haben und nun mehr tun sollen, als zehn Jahre und länger ohne weitere Entwicklungsperspektive auf die Rente zu warten (frühe Ansätze: Migros/Schweiz, Siemens Erlangen).

14. Entwicklung und Durchführung vielfältiger gesundheitsorientierter Maßnahmen aus dem Führungs-, Entspannungs- und Bewegungs-, sowie Krankheits-Früherkennungsbereich. Teilweise werden derartige Angebote von Betriebsexternen durchgeführt, was auch für Präventionsmaßnahmen gegen Burn-out und Mobbing sowie Konfliktmanagement gilt. Für Branchen, in denen kaum Gesundheitsförderungsimpulse seitens des Betriebes zu erwarten sind (Beispiel kleinbetriebliche Gastro), wäre laut Indigho-Projektgruppe an den Aufbau von individuellen Gesundheitsressourcen in der Ausbildung und durch Beratung für Quereinsteiger*innen zu denken ${ }^{20}$.

15. Zusatzangebote im Concierge-Bereich zur Entlastung Beschäftigter von haushaltsnahen Aufgaben (z.B. über die Firma buchbarer Wäscheservice, Kantinenessen zum Mitnehmen, Putzservice o.Ä.).

Die selektive Auflistung zeigt, dass nur ein kleinerer Teil der Maßnahmen „speziell für Ältere“ definiert ist (wie Freischichten und Altersteilzeit), ein Großteil jedoch allen Belegschaftsgruppen unabhängig vom kalendarischen Alter zugänglich ist (was im Bereich „Fortbildungen auch für Ältere" keine Selbstverständlichkeit darstellt und auch von den älteren Belegschaftsangehörigen nicht überall mit offenen Armen begrüßt wird $\left.{ }^{21}\right)$. Das AGG erschwert es hier offenbar nicht nur, Ältere zu diskriminieren, sondern ebenso auch Jüngere, weshalb Maßnahmen speziell für Ältere in den Betrieben oftmals kritisch gesehen werden ${ }^{22}$. In Untersuchungen des repräsentativen Betriebspanels des IAB gaben stets um die 20\% der Unternehmen an, Maßnahmen dezidiert für Ältere vorzuhalten, was aber zugleich auch bedeutet, dass die Nennung pro Einzelmaßnahme (und damit deren jeweiliger Verbreitungsgrad) noch geringer ist. Laut EBA-Studie ${ }^{23}$ machen die anderen Firmen nicht etwa gar nichts, sondern sie lassen ihre Personalmaßnahmen lediglich nicht unter den Labeln „Altersmanagement" oder "für Ältere" laufen, was letztlich auch als diskriminierend empfunden werden könnte. Hier ist auf eine interessante Studie von Henner Hentze et al. zu verweisen, in der Personen befragt wurden, die innerhalb der vorausgegangenen zwei Jahre in den Ruhestand eintraten ${ }^{24}$. Diese sprachen sich für kompetenz- und erfahrungsbasierte Tätigkeiten aus anstelle klassischer Schon-Arbeitsplätze oder Maßnahmen „für Ältere“25. Den Befragten war es ferner ein Anliegen, ihr Wissen innerhalb ihres Betriebes weitergeben zu dürfen, sowie dass es wertschätzende Verabschiedungsri- 
tuale im Betrieb geben solle. Hierin ist ebenso ein wichtiger Grund zu sehen, warum die Deutsche Rentenversicherung im Zuge der Verbesserung der Arbeitgeberberatung im demografischen Wandel ein "Generationenmanagement im Arbeitsleben (GeniAL)" etabliert hat (auch und vor allem mit Fokus auf dem Mit- und Nebeneinander der Alterskohorten) ${ }^{26}$. Mit Blick auf die Implementationsschwierigkeiten im Modell der Arbeitsfähigkeit setzen Conrads et al. (2014) für die Metallindustrie auf sozialpartnerschaftliche Betriebsvereinbarungen, die neben Maßnahmen eine breite Aufklärungs- und Informationskampagne enthalten (Schlagwort: „Demografiearena" mit arbeitsplatznahen Schulungen eines Großteils der Belegschaften auf dem Werksgelände). ${ }^{27}$

Zum Altersmanagement gehört auch noch die Frage der Ruhestandsvorbereitung mit Unterstützung bei der Statuspassage in Rente und der Suche nach neuen Aufgaben, die idealerweise schon vor dem Renteneintritt eingeleitet werden sollten.

\section{Regelungsbeispiele aus Tarifverträgen und Betriebsvereinbarungen im Lichte des wissenschaftlichen Forschungsstandes}

Im nachstehenden, notwendigerweise selektiven Kapitel werden einige bekanntere und weniger bekannte Branchenbeispiele herausgegriffen, und hierbei auch auf die Schwierigkeiten und Herausforderungen bei deren Durchsetzung verwiesen. Aus wissenschaftlicher Sicht ist es oftmals interessanter, wie Mindeststandards guter Arbeit in schwierige Branchen und Tätigkeitsbereiche gebracht werden konnten, als gute Praxisbeispiele lukrativer Branchen mit hohem Fachkräfteanteil und etablierten Mitarbeitervertretungen aufzulisten.

Einzelansätze zur Verankerung von Aspekten guter Arbeit in Tarifverträgen existieren seit den 1970er-Jahren, und manche Themenfelder wie Mitbestimmung, Schutz vor Machtmissbrauch und sexueller Belästigung wurden bereits um 1900 diskutiert $^{28}$. Explizit unter dem Terminus „Demografietarifverträge“ werden zumeist genannt: Metall 2006 (im NRW-plusGebiet) ${ }^{29}$; Chemie 2008, 2012 und 2016; Verkehr (Entwurf für Hamburg 30 und Schleswig-Holstein 2010); Öffentliche Wasserwirtschaft 2012; Uni-Kliniken Freiburg, Heidelberg, Tübingen und Ulm 2013; ÖPNV 2014; Papierindustrie 2014; Genossenschaftsbanken 2014; Holz und Kunststoff verarbeitende Industrie 2016; Energiewirtschaft 201631.

Um Spielraum für einzelbetriebliche Besonderheiten und deren Ausgestaltung zu lassen, geben qualitative Tarifverträge einen Handlungsrahmen vor (im Sinne der sog. „weichen Koordinierung“), der dann in den Betrie- 
ben mit Leben gefüllt werden muss (dezentrale Konkretisierung). Hierzu bedarf es oftmals Wahloptionen, um betrieblichen Besonderheiten gerecht werden zu können.

Prozesstarifverträge stoßen Prozesse an und sind getragen von der Hoffnung auf eine Entwicklung Lernender Systeme und weiterer Schritte im Zeitverlauf. Hier lässt sich ein Schwenk von der Frage „Was soll gemacht werden?" (mit Fokus auf Handlungsfeldern und Instrumenten) hin zu „Wie soll Demografie-bezogenes Denken und Handeln im Sinne einer längerfristigen Personalpolitik initiiert und verankert werden?" konstatieren.

Tarifverträge wirken nicht nur bezüglich der Mitgliedsunternehmen, sondern strahlen oftmals auch auf weitere Firmen aus, die zumindest Teile davon übernehmen (Impulsfunktion).

Große Unternehmen haben oft eigene Tarifverträge und Konzernbetriebsvereinbarungen (wie Post, Bahn, Einzelhandelsketten), wobei nicht alle Konzernteile jeweils auch alle Regelungen übernehmen (Beispiel Deutsche Bahn).

In kleineren und mittleren Unternehmen finden wir eine Reihe von Einzelmaßnahmen, die jedoch nur selten systematisch miteinander verzahnt sind. Die Maßnahmendichte korreliert hier mit der betriebswirtschaftlichen Situation und den qualitativen wie quantitativen Personalressourcen.

Folgende Regelungsbereiche sind festzustellen:

- Bestandsaufnahmen („Altersstrukturanalyse“ oder „Demografieanalyse“, auch nach Abteilung und Qualifikationsgruppe; Tätigkeitsanalysen und Gefährdungsbeurteilungen ${ }^{32}$; Qualifikationsanalysen inklusive des künftig ermittelten Bedarfs).

- Vorgaben zur Kooperation und Deutung der Befunde (einvernehmlich durch Betriebsleitung, Personalabteilung und Betriebsrat; Datenbereitstellung).

- Verbesserung von Führung, Arbeitsorganisation und Arbeitsbedingungen, Ergonomie (bspw. Lärmschutz, Beleuchtung).

- Finanzierungsgrundlagen der Maßnahmen (z.B. Demografiefonds, Lebenszeitkonto, Einzahlmodalitäten, mögliche Mittelverwendung, Fragen der Mitnahmemöglichkeit von Ansparfonds - sog. „Portabilität“ -, bis hin zur Vererbung angesparten Kapitals); hierunter fallen auch Ausstiegsklauseln unter bestimmten Voraussetzungen (Exit-Optionen).

- vorgesehene Nutzungsvarianten und Anspruchsvoraussetzungen, beispielsweise die Regelung von Entlastungszeiten (wie Freischichten, Altersteilzeit - auch im Blockmodell, bezahlte Freistellung für Gesund- 
heitstage) bei bestimmten Tätigkeiten/Arbeitsvarianten und oder ab einem bestimmten Alter - teilweise auch mit nach Alter gestaffelten Ansprüchen $^{33}$ (z.B. Busfahrer*innen, bei Nachtschichten und im Vollkonti-Schichtmodus; teils nach kalendarischem Alter geregelt und teils nach der Dauer der Ausübung eines bestimmten Tätigkeitsformats). Eine Besonderheit der Entlastungsvereinbarung stellen in den letzten Jahren erkämpfte Vereinbarungen in Krankenhäusern für den Fall einer bestimmten personellen Unterbesetzung dar, denen zufolge dann Abteilungen geschlossen werden müssen ${ }^{34}$.

- zur ortsflexiblen Arbeitszeit bei der Telekom (Regelungen für Anwesenheit am Arbeitsplatz und für mobiles Arbeiten mit „doppelter Freiwilligkeit“ für die Arbeitgeber- und die Arbeitnehmerseite ${ }^{35}$.

- finanzieller Ausgleich für Phasen mit reduzierter Arbeitszeit wegen Erziehung und Pflege, Weiterbildung oder Sabbatical, aber auch zur Entgeltsicherung bei reduzierter bzw. modifizierter Leistungsfähigkeit (und beispielsweise einem Wechsel vom besser bezahlten und anspruchsvolleren Akkord-Modus in den Zeitlohn-Bereich) ${ }^{36}$. Zu finden sind auch Regelungen für die Anrechnung von Familienzeiten, die dann ganz oder teilweise wie Betriebszugehörigkeitszeiten gerechnet werden ${ }^{37}$.

- Regelungen zum Einbezug Leistungsgewandelter mit Faktor-Anrechnung im Gruppenakkord ${ }^{38}$.

- Umsetzungshinweise und Unterstützungsangebote bei der Realisierung tarifvertraglicher Regelungen.

Feststellen lassen sich bestimmte Trends:

- Weg von Vorruhestandsoptionen und Frühverrentung hin zu Entlastungszeiten entsprechend einem bestimmten Alter und/oder Tätigkeitsbereich (Beispiel Verkehrsbetriebe).

- Öfters finden sich Optionen ähnlich reduzierter Vollzeit im Umfang von $80 \%$, wobei die Arbeitszeitreduktion teilweise von Arbeitgeberseite kompensiert wird. In der BA erfreut sich das 9/10tel-Arbeiten (mit etwa 20 mehr freien Tagen pro Jahr) einer gewissen Beliebtheit.

- Erhebliche Unterschiede zeigen sich je nach Branche, beispielsweise im Vergleich von Gastronomie und Einzelhandel mit Chemie und Elektro, sowohl was Belegschaftszusammensetzungen und Betriebsgrößen anbelangt, aber auch bezüglich der Alterszusammensetzung und des Frauen- und Teilzeitanteils, der Frage von Betriebskontinuität oder diskontinuierlichen Erwerbsbiografien, wie auch der unterschiedlichen Stärke von Betriebsräten (so überhaupt vorhanden). Während in der 
Gastronomie und im Einzelhandel viele soziale Kompetenzen wie Kundenorientierung nötig sind, aber nicht unbedingt lange Ausbildungen, setzen fordistische Branchen eher auf formale Ausbildungsgänge und möglichst langfristige Betriebszugehörigkeit, kennen aber keine Jugendlichkeitserwartung. In ersteren Branchen arbeiten mehr jüngere Menschen, mehr Frauen und mehr Teilzeitbeschäftigte, es gibt mehr Personen mit diskontinuierlichen Lebensläufen und insgesamt schwächere Betriebsräte. Aus Arbeitgebersicht dominiert die Vorstellung einer vergleichsweise leichten Ersetzbarkeit des Personals. Anders stellt sich die Situation in den fordistischen Segmenten dar: Hier dominieren idealerweise Berufskontinuität und Vollzeitjob zumindest in den Produktionssegmenten, hier sind Fachkräfte schwer zu ersetzen und Berufserfahrung stellt einen eigenen Wert dar und führt zur Wertschätzung älterer Beschäftigter ${ }^{39}$. Betriebsräte sind hier verhältnismäßig stark, was u.a. auch an stabileren und größeren Firmen liegt. Je größer der Betrieb, umso ausdifferenzierter und fachlich versierter ist i.d.R. auch die Personalabteilung. Je nach Branche dominieren eher KonsensOrientierung (Chemie) oder Konfrontation (Metall).

- Interessant zu sehen sind tarifpolitische Auseinandersetzungen in Branchen, bei denen Lohn und Arbeitsbedingungen nicht dem freien Markt überlassen, sondern zumindest auch politisch festgesetzt werden wie im Krankenhaus- bzw. Pflegebereich. Offenbar folgen hier einer zunehmenden öffentlichen Wertschätzung Taten erst dann, wenn entsprechender Druck aufgebaut wird. Fachkräfte zeigen sich hier nicht mehr länger gewillt, widrige Arbeitsbedingungen und personelle Unterbesetzung hinzunehmen, zumal letztere mit hohen Risiken für die Gepflegten einhergeht (Stichwort: „gefährliche Pflege"). Fortschritte sind zwar festzustellen und schlagen sich auch in Tarifverträgen nieder, doch macht sich Ernüchterung breit, wenn man betrachtet, wie lange kritische Pflegebedingungen schon öffentlich diskutiert werden. ${ }^{40}$

Verlässt man die Perspektive der unternehmensübergreifenden Tarifverträge und begibt sich auf die einzelbetriebliche Ebene, so stößt man teils auf Konkretisierungen tarifvertraglicher Rahmenvorgaben (inklusive Optionsklauseln), und teils auf unternehmensindividuelle bzw. auch auf an Tarifverträge angelehnte Maßnahmen (selbst bei nicht tarifvertraglich gebundenen Firmen). 
Arbeitszeitregelungen spiegeln einen Spagat zwischen betrieblichen und Mitarbeiter"innen-Bedürfnissen. Hier zeigt sich ein Flexibilisierungstrend in zwei Richtungen: Einerseits sind Entgrenzungen festzustellen, etwa was Ladenöffnungszeiten, Events, ÖPNV, und Care-Aufgaben anbelangt, und andererseits ergeben sich hieraus neue Möglichkeiten bedürfnisorientierter Arbeitszeitmodelle. Wenngleich immer noch auf niedrigem Niveau, geht der Trend zumindest bei großen Betrieben bzw. Unternehmen in Richtung Unterstützung bei Pflegeaufgaben und Kinderbetreuung, und zur Mitarbeiter*innen-Anbindung während Familienpausen zum Erhalt betriebsbezogenen Wissens und aktueller Qualifikation. Ein wichtiges Thema ist die Kinderferienbetreuung, die auch aus Demografiefonds (mit)finanziert werden kann. Die Bundesagentur für Arbeit unterhält im Rahmen ihres sog. „Lebensphasenorientierten Personalpolitikmodells“ einen Organisationsservice Kinder \& Pflege (OKiP) in Kooperation mit der Arbeiterwohlfahrt ${ }^{41}$ und reagiert damit zugleich auf den Sachverhalt, dass immer häufiger betagte Eltern und Kinder- wie Enkelgenerationen an unterschiedlichen Orten wohnen und daher letztere kaum in der Lage sind, Pflege am Wohnort der Eltern oder Großeltern zu leisten. Ein weiterer Trend, der diesbezüglich der Betrachtung wert ist, besteht darin, dass heute mehr Frauen erwerbstätig sind als früher und es zudem wegen vermehrter Ein-Kind-Familien immer weniger Onkel und Tanten gibt, sodass das innerfamiliäre Kinderbetreuungspotenzial durch Großeltern u.a. Verwandte enorm geschrumpft ist, weshalb es betrieblicher Unterstützung mit Flexibilitätspuffern und kreativen Lösungen bedarf, wovon nicht nur Alleinerziehende profitieren.

- In Zusammenhang mit der Notwendigkeit der Synchronisierung lebensphasentypischer Herausforderungen mit der Erwerbsarbeit sowohl in einkommensbezogener wie in arbeitszeitlicher Hinsicht bieten Lebenszeitkonten vielfältige Möglichkeiten. Im Idealfall gibt es hierzu eine Anschubfinanzierung durch das Unternehmen und ein Ansparen der Konten in Geld und Zeiteinheiten durch die Beschäftigten. Über kontinuierliches Ansparen sollten sich dann Betreuungs- und Pflegezeiten, aber auch Weiterbildungskorridore finanzieren lassen.

- Aus dem Bereich der Chemie zeigen integrierte Safety-Health-Environment-Ansätze in Richtung mehr Achtsamkeit im idealerweise pfleglichen Umgang mit Ressourcen jeglicher Art. Aus Demografietöpfen lassen sich u.a. Massage- und Fitnessangebote, Beteiligungen an Firmenläufen und bisweilen sogar Nutzungsmöglichkeiten von Sportstätten für Angehörige der Beschäftigten mitfinanzieren. Hinsichtlich der Lebenszeitkonten lautet eine Erkenntnis, dass Jüngere hierfür leichter zu 
gewinnen sind, wenn deren Nutzung nicht erst im reifen Erwerbsalter möglich ist, sondern auch schon früher (etwa bei Kinderbetreuungsund Pflegeaufgaben, Weiterbildungsfreistellungen u.Ä). Damit verbunden ist eine Fokuserweiterung von der späten Erwerbsphase hin zum gesamten Lebenszyklus und hin zu Vereinbarkeits- u. a. Diversityfragen, die auch das Arbeitgeberinteresse einer Ausstrahlwirkung nicht nur für Ältere bedienen ${ }^{42}$.

- Die ganze Lebensspanne in den Blick nimmt auch der Bahntarifvertrag zwischen DB-Konzern, EVG und dem Arbeitgeberverband MoVe (2013/2016). Hier finden sich Überlegungen und Regelungen zur Rekrutierung von Auszubildenden, zur Lebensphasen- und Lebenslaufgerechtigkeit mit Kinderbetreuung und Pflegeunterstützung, bis hin zu Altersfreischichten und einer „besonderen Teilzeit im Alter" und klar geregelten Angeboten für Leistungsgewandelte. Angestrebt wird eine Trias von gestärkter Arbeitgeberattraktivität, erhöhter Mitarbeiterzufriedenheit und geförderter Demografiefestigkeit. Das Unternehmen gibt hier ein Commitment ab zur dauerhaften Belegschaftsbindung und einer Verantwortungsgemeinschaft für die Beschäftigungsfähigkeit der Mitarbeiter"innen. Hierzu gehören u.a. konzerninterne Jobbörsen und eine eigene Zeitarbeitsfirma, eine Sicht auf externe Zeitarbeiter"innen als potenzielle künftige Dauermitarbeiter*innen sowie wissenschaftsgestützte Profi-Angebote zur betrieblichen Gesundheitsförderung. Konzerneigene Umsetzungsunterstützer"innen und Konfliktmediator"innen helfen bei der Realisierung. Bildungsangebote zur Kommunikation in Krisensituationen (etwa gegenüber aufgebrachten Kunden) sowie zum Erhalt der psychischen Gesundheit und zur Stärkung körperlicher und geistiger Fitness runden die Personalmaßnahmen ab. Deren Wirkungen werden evaluiert ${ }^{43}$.

- Ein interessantes Beispiel für die Sicherung von Mindeststandards guter Arbeit bei gleichzeitiger Vermeidung von Dumping-Konkurrenz bei Ausschreibungen sind die Regelungen für Bodenverkehrsdienste am Münchner Flughafen mit arbeitsmedizinischen und qualifikatorischen Mindeststandards ${ }^{44}$. Beim Gepäckmanagement werden beim Be- und Entladen von Flugzeugen pro Arbeitskraft und Schicht bis zu $1500 \mathrm{Ge}-$ päckstücke im Gesamtgewicht von ca. 22 Tonnen händisch bewegt, und das auch noch teilweise in gebückter Haltung. Somit handelt es sich um einen Tätigkeitsbereich, der nahezu klassisch ist für verschleißintensive und alternskritische Tätigkeiten, die ob überschaubarer qualifikatorischer Anforderungen gut an externe Dienstleister"innen vergeben werden könnten, unter denen derjenige mit dem günstigsten Preisangebot den Zuschlag erhalten würde. Der niedrigste Preis 
dürfte jedoch mit hoher Wahrscheinlichkeit nur mit gering entlohnten Beschäftigten und einem entsprechend hohen Anteil an Personen mit Migrationshintergrund und einer allenfalls schwachen Mitarbeitervertretung zu erreichen sein. Mithilfe wissenschaftlicher Unterstützung gelang es am Münchner Flughafen, über Regelungen hinsichtlich arbeitsmedizinischer und qualifikatorischer Mindeststandards Dumping-Konkurrenz zu vermeiden und für ein Minimum an guten Arbeitsbedingungen zu sorgen ${ }^{45}$.

- Nicht jeder Betrieb braucht für alle denkbaren Bedürfnisgruppen Regelungen, sondern kann sich sein individuelles Maßnahmen-Portfolio nach Bedarf zusammenstellen. Wichtig dabei wäre, dass Gruppenbedürfnisse nicht gegeneinander ausgespielt werden (was beispielsweise dann der Fall wäre, wenn „Familienfreundlichkeit“ zulasten kinderloser Personen ginge), und dass Unterschiede nicht starr wahrgenommen und betreffende Personen(gruppen) nicht in Schubladen eingeteilt werden. Bedeutsam ist hierbei, wie mit derartigen Spannungslinien bzw. Konflikten betriebsintern umgegangen wird, und wie Demokratiegrundlagen, wie betrieblicher Gesundheitsschutz, wie Minderheitenschutz betriebsintern kommuniziert und geregelt werden. Derlei Fragen verweisen wiederum darauf, dass Herausforderungen, mit denen die allermeisten Unternehmen eigenverantwortlich im Alltag gut zurechtkommen, nicht im Tarifvertrag verankert werden müssen. Hier wäre das Thema „Delegitimation durch Überregulierung“ angesprochen (in Analogie zum „Veggie Day“ der Grünen, der nicht an inhaltlichen Fragen gescheitert ist, sondern daran, dass sich Menschen ungerne bevormunden lassen; analoge Beispiele richten sich auf Sport und Bewegung, die Wahl des Verkehrsmittels zur Arbeit, und bisweilen sogar das Nutzen ergonomischer Hebe- und Tragehilfen und/oder Sicherheitsvorschriften. Maßnahmen zur Erhöhung der Akzeptanz von Aktivitäten des betrieblichen Gesundheitsmanagements oder des Demografiemanagements sind daher sehr bedeutsam für eine gute Zielerreichung $\left.{ }^{46}\right)$.

- Schwierig bis kaum zu regeln sind Bereiche wie in der überwiegend kleinbetrieblichen Gastronomie-Branche, die gekennzeichnet ist von untypischen Arbeitszeiten (auch abends und am Wochenende), kundeninduzierten und damit volatilen Nachfrageströmen und Stoßzeiten, Multitasking-Anforderungen, vielen Teilzeit- und geringfügig Beschäftigten, einem hohen Gastarbeiter"innen- bzw. Migrant"innenanteil, einer hohen Konkurrenz und zahlreichen Marktein- und -austritten entsprechender Firmen, einer geringen Mitarbeiterbindung an die Firmen als Strukturprinzip und allgemein wenig formalen Qualifikations- 
anforderungen für die Ausübung der Tätigkeit. Impulse zur Personalentwicklung müssten hier vor allem von außen kommen, z.B. durch Ausbildung, Beratung, Dachverbände ${ }^{47}$.

\section{Zusammenfassende Deutung zum Stand der Umsetzung betrieblicher Demografiepolitik}

Gezeigt werden sollte, dass es weiterhin alters- und alternskritische Tätigkeitsfelder mit unterschiedlicher körperlicher, psychischer und mentaler Beanspruchung gibt und auch künftig geben wird. Diese gilt es mit den Dimensionen des „Hauses der Arbeitsfähigkeit“ und wissenschaftsgestützten wie unter Beteiligung von Mitarbeitervertretungen und Mitarbeiter"innen entwickelten Handlungsansätzen zu analysieren und soweit möglich zu humanisieren. Eine eingeschränkte Arbeitsfähigkeit im reiferen Erwerbsalter ist dabei wesentlich als Folge widriger Arbeitsbedingungen und in früheren Erwerbsphasen unterbliebener Investitionen in Humanressourcen zu verstehen. Demografieorientierung erstreckt sich neben Fragen im Kontext des Älterwerdens im Betrieb auch auf weitere Diversity-Fragen wie die Unterstützung von Care-Aufgaben, interkulturelle Kooperation und behinderungsgerechte Arbeitswelt- und Arbeitsplatzgestaltung, sowie Inklusionsanliegen jeglicher Couleur. Die Überwindung des wissenschaftssegmentären Feldes der Alters- und Alternsforschung und deren Erweiterung hin zu vielfältigen HR-Aufgaben und Erwerbsbiografiegestaltung auch unter Einbezug typischer Lebensphasen, Fortbildungs- und Umschulungsaufgaben und Gesundheitserhalt, also zu Diversity-Management im weiteren Sinne, wird den betrieblichen Herausforderungen eher gerecht. Hierdurch wird ein Gegeneinander-Ausspielen unterschiedlicher Altersgruppen, kontinuierlich und temporär Beschäftigter, divergenter Geschlechter, sowie von Personen mit und ohne aktuelle Verantwortung für abhängige Angehörige vermieden.

Aus wissenschaftlicher Sicht wird deutlich, dass betriebsindividuelles Lernen über mehrere Stufen hin zu einem integrierten lernenden System wünschenswert wäre und prozessualer Unterstützung inklusive einer Informations- und Weiterbildungsstrategie bedarf. Der Unterstützungsbedarf gilt umso mehr, je kleiner die Unternehmen und je geringer die quantitativen und fachlichen Personalmanagement-Ressourcen im jeweiligen Betrieb sind. Besonderes Augenmerk benötigen Tätigkeitsbereiche, die mit hohen Arbeitsbelastungen und zugleich niedrigen qualifikatorischen Voraussetzungen einhergehen. Von Relevanz ist dies insbesondere dann, 
wenn die jeweilige Branche eher wenig lukrativ ist, und deshalb kaum finanzielle Mittel für Personalmaßnahmen zur Verfügung stehen.

Beratungs- und Fachleutenetzwerke und entsprechende Institutionen (wie BAuA/INQA, ddn) sind gefordert, bei der Erschließung und dem Aufbau demografiebezogenen Managementwissens mitzuhelfen, und dabei die Besonderheiten von Branchen und Betriebsgrößen mit im Blick zu haben. Die Verantwortung für eine Kooperation zwischen den Personalfachleuten von Arbeitswissenschaftler*innen bis Arbeitsmediziner*innen auf der einen und den betrieblichen Akteuren auf der anderen Seite ist sowohl Bring- wie Holschuld. An Modellen und Instrumenten mangelt es nicht, wie die BAuA-Toolbox, das Haus der Arbeitsfähigkeit, PsyGA, der Gewerkschaftsdiskurs um gute Arbeit, WAI/ABI, Altersstrukturanalysen, Arbeitsplatzstrukturanalysen, Gefährdungsbeurteilungen, Qualifikationsanalysen, Diversity-Zirkel, Analysen von Fluktuations- und Abwesenheitszahlen zeigen. Für Tarifkommissionen und die in diesen Tätigen besteht die Herausforderung jedoch darin, dass die meisten Instrumente erst bei professioneller Anwendung und Deutung der damit zutage geförderten betriebsbezogenen Zahlen sowie bei einer hohen Mobilisierung und Beteiligung der Beschäftigten nennenswerte Wirkungen entfalten. Entsprechend zeichnet sich ein Trend zu Prozesstarifverträgen ab, d.h. neben den Zielen müssen die Wege dorthin konkretisiert und die Umsetzungsakteure instruiert, unterstützt und begleitet, sowie die Beschäftigten auf breiter Basis in ein Kommunikationsmanagement eingeflochten werden. Die Branchen- und Firmenbeispiele machen deutlich, dass dies dann erleichtert wird, wenn es Unterstützung von der Firmenleitung und eine beteiligungsorientierte Managementkultur gibt, die Beschäftigte als „Experten ihrer Arbeit“ betrachtet und mit ihnen einen anerkennenden Erfahrungsaustausch betreibt ${ }^{48}$, wenn Antreiber*innen sowohl aus der Firmenleitung wie aus der Personalabteilung und dem Betriebs- oder Personalrat kommen, und wenn bereits Funktionsabteilungen bestehen, an die neue Prozesse (inklusive Ausschüsse und Zirkel) angedockt werden können, und die bereits eine gewisse Prozesserfahrung haben. Idealerweise besteht zwischen den betrieblichen Akteuren trotz unweigerlicher Interessengegensätze und Konflikte in manchen Bereichen ein Mindestmaß an Kooperationsfähigkeit und Vertrauen.

Die im Schrifttum dokumentierten guten Praxis-Beispiele, insbesondere was systemisch integrierte Handlungsansätze und Maßnahmenbündel betrifft, entstammen im Regelfall Großunternehmen mit gut ausgebildeten Fachkräften, an deren Gewinnung und Bindung auch den Firmenleitungen gelegen ist. Geht man nun der Frage nach, wie weit das Demografiemanagement in mittelgroßen Betrieben von 50 bis 1000 Beschäftigten ge- 
diehen ist, ist man auf branchenbezogene Analysen mit zumindest auch qualitativen Analyseteilen verwiesen. Nimmt man die Chemie als Vorzeigebranche mit schon früher Sensibilität für Demografiefragen, so darf angenommen werden, dass Befunde aus diesem Segment zumindest dahingehend Gültigkeit beanspruchen können, dass andere Branchen auch nicht weiter sein dürften. Feststellen lässt sich für mittelgroße Betriebe der Chemischen Industrie:

- Der demografische Wandel ist in den Betrieben zumeist bereits spürbar, beispielsweise in dem Sinne, dass sich nicht mehr automatisch genügend Lehrstellensuchende bewerben und der Betrieb nur noch auswählen muss, wie das früher oft der Fall war, weshalb nun gezielte Rekrutierungsmaßnahmen über mehrere Kanäle entwickelt und gepflegt werden müssen. Problemdruck alleine führt jedoch noch nicht automatisch zu proaktivem Handeln; hierzu bedarf es der Anstöße, Gelegenheitsstrukturen und Umsetzungsprotagonist"innen, sowie gerade in kleineren und mittelständischen Unternehmen bisweilen auch externer fachlicher Hilfe. ${ }^{49}$

- Ein in sich integriertes Demografiemanagement-System ist sehr selten; stattdessen dominieren Einzelmaßnahmen, die sich von Betrieb zu Betrieb unterscheiden, und insgesamt stark pfad- und ressourcenabhängig sind. ${ }^{50}$

- Sowohl der Personalaufbau wie der Personalabbau werden zur demografischen Gestaltung der Belegschaft genutzt.

- Demografieverträgen kann eine Anstoß- und Begleitwirkung („Nudging“) zugesprochen werden.

- Gesundheitsförderansätze sind teilweise beliebig und selten tätigkeitsrückgebunden. Verbreitet erscheint die Haltung zu sein, dass jegliche Maßnahme irgendwie helfe. Jenseits primärpräventiver Bemühungen zeigen sich kaum Angebote für gesundheitlich bereits Angeschlagene. Kritisch betrachtet ließen sich manche Maßnahmen auch als Symbolpolitik bezeichnen.

- Unternehmen kümmern sich im Mittel immer noch (zu) wenig um Care-Fragen. Diese werden meist als Privatsache eingestuft, oder aber als "quantitativ zu unerheblich, um sich damit zu befassen." Auch wenn zeitgleich oft nur einige wenige Personen mit Pflegekonstellationen betraut sind, würde ein erweiterter Blick auf einen Zehnjahreszeitraum zeigen, dass ein ganz erheblicher Teil der 50plus-Beschäftigten irgendwann Pflegeaufgaben managen muss. 
- Demografiefonds ${ }^{51}$ werden nicht nur für Ältere ausgegeben (z.B. für Freischichten), sondern auch im Sinne des o.g. Lebensphasen- bzw. Diversity Managements verwendet.

- Eine Humanisierung von Arbeitsformaten erfolgt oftmals bei der Inbetriebnahme neuer Anlagen; allerdings sind es oft die Älteren, die noch an den älteren Produktionsstraßen arbeiten.

- Eine fachliche externe Unterstützung wird meist erst ab einem mittleren Personalmanagement-Niveau gezielt gesucht und miteinbezogen; darunter dominiert eine (meistens reaktive) Einzelfallbehandlung von Personalfragen, die meist im Konsens von Betriebsleitung, Personalmanagement und Betriebsrat entscheidet, aber kein lernendes System entwickelt.

- Fachliche Impulse gehen einmal von der Betriebsleitung, einmal von der Personalabteilung und einmal vom Betriebsrat aus - je nach Erfahrung, Netzwerkkooperationen und fachlichem Hintergrund.

- Auf allen o.g. drei Seiten herrscht eine gewisse Vorsicht, Maßnahmen mit ungewisser Erfolgswahrscheinlichkeit anzustoßen, weil damit jeweils Delegitimationsrisiken einhergehen. Das gilt auch für in der Umsetzung komplizierte Maßnahmen, beispielsweise mit Einzelfallentscheidungen darüber, wer in den Genuss bestimmter Maßnahmen kommt und wer nicht. Universalmaßnahmen sind demgegenüber beliebter.

- Ältere sind in Maßnahmen zur Weiterbildung und zur Gesundheitsförderung mit eingebunden, es finden sich - abgesehen von Freischichten - jedoch nur wenig spezifische Maßnahmen exklusiv für Ältere, nicht zuletzt aus Angst vor AGG-Verstößen, falls Maßnahmen als einseitige Bevorzugung einer Altersgruppe ausgelegt werden würden.

- Körperorientierte Maßnahmen, beispielsweise Massagen gegen Fehlbelastungsstörungen, sind deutlich verbreiteter als salutogenetische bzw. präventive Maßnahmen im psychischen Bereich, beispielsweise zur Stressbelastungsreduktion.

- Auffangklauseln, die dann greifen, wenn keine kreativere betriebliche Lösung gefunden wurde, definieren Mindeststandards und, prozessual betrachtet, erste Entwicklungsstufen eines Demografiemanagements.

- Kranken(rückkehr)gespräche („Willkommensgespräche“) und Betriebliches Eingliederungsmanagement sind nicht überall verbreitet und bei Belegschaftsangehörigen auch nicht überall akzeptiert (insbesondere bei psychischen Belastungen im Arbeitssetting). Ungünstige Interaktionsbeziehungen und psychische Belastungen scheinen ein Tabu-Thema zu sein und werden i.d.R. erst angegangen, wenn ein Mindestmaß an 
gegenseitigem Vertrauen zwischen der betreffenden Person und weiteren betrieblichen Akteuren besteht.

- Altersstrukturanalysen gehen mitunter über reine Zahlenkonglomerate (ohne strategische Deutung und Maßnahmenentwicklung) nicht hinaus. Gelegentlich wurden Zahlen zwar erfasst, aber nicht an den Betriebsrat weitergegeben.

- Selbst in Vorzeigebetrieben findet man Personalverantwortliche, die sich außerstande sehen, ihren im Branchenvergleich (zu) hohen Krankenstand zu deuten.

- Mitunter stößt man auf betriebliche Arbeitsformate für Ältere und/ oder Leistungsgewandelte, die nicht stigmatisierend sind und gut angenommen werden. Diese entsprechen den in der Studie von Henner Hentze in Münster ermittelten Wünschen nach einem kompetenzbasierten Arbeiten anstelle der Schon-Arbeitsplätze ${ }^{52}$.

Wissenschaftliche Einschätzungen schwanken bei der Betrachtung kleiner bis mittelgroßer Firmen zwischen dem Lob für die beobachtbar große und kreative Vielfalt von Einzelmaßnahmen ${ }^{53}$ trotz der zusammenfassenden Diagnose eines empirischen „Stückwerks“, und der deshalb überwiegenden Kritik an einer mangelnden systematischen Verzahnung innerhalb eines strategischen Rahmens ${ }^{54}$.

Mit Colin Crouch 2019 lassen sich parallel Verbesserungen und Verschlechterungen in der Arbeitswelt feststellen, d.h. es finden sich sowohl positive Regulierungs- und Gestaltungsprozesse wie neue Formen der Reprivatisierung und Ausbeutung. In belastenden Branchen wird oft mit atypischen Beschäftigten (wie in Teilzeit arbeitenden Frauen, Leiharbeiter"innen, Mini-Jobber"innen ...) gearbeitet, und dadurch der Erholungsaufwand privatisiert und externalisiert.

\section{Tarifpolitische Perspektiven und Handlungsempfehlungen}

Es konnte gezeigt werden, dass eine älter werdende und vielfältigere Beschäftigtenstruktur angesichts von kleiner werdenden nachwachsenden Erwerbstätigenjahrgängen einen personalpolitischen Handlungsbedarf aufwirft. Dies gilt umso mehr, je verschleißintensiver, alters- und alternskritischer die jeweiligen Tätigkeiten sind. Tarifverträge und Betriebsvereinbarungen stellen, neben rechtlichen Vorgaben (z.B. Arbeitszeitgesetz, Betriebsstättenverordnung, Betriebsverfassungsgesetz u.Ä.) und bilateraler deliberativer Aushandlung, wichtige Instrumente zur Festlegung von Arbeits- und Rahmenbedingungen dar. 
Das Thema des Alters und des Alterns in Betrieben steht nicht alleine, sondern in Konkurrenz zu Fragen wie Vereinbarkeit, Arbeitszeitflexibilität, Karrieren nicht nur unilinear und hierarchisch, sondern auch mit flexibleren Verläufen je nach Lebensphase entsprechend dem Modell eines Klettergerüsts, in dem man auch mal zur Seite und nach unten klettern, oder eine gewisse Zeit auf einer Stufe verharren kann. Untersuchungen zu Veränderungen zwischen den Generationen und deren Erwartungen zeigen für die beiden jungen Kohorten $\mathrm{Y}$ und $\mathrm{Z}$, dass hier erweiterte Forderungen hinsichtlich Work-Life-Balance, vollzeitnaher Teilzeit und einer egalitäreren Verteilung reproduktiver und erwerbszentrierter Arbeit zwischen den Geschlechtern herrschen ${ }^{55}$. Entsprechend sollten Entlastungsmöglichkeiten nicht nur gegen Ende des Erwerbslebens, sondern flexibel je nach Lebensphase ermöglicht und finanzierbar gemacht werden. Demografiefonds sind diesbezüglich eine gute Sache, und Freistellungsmöglichkeiten auch schon in jüngeren Jahren (z.B. für Weiterbildung, Kinderbetreuung oder Pflege, aber auch für Sabbaticals oder Hausbau) erleichtern lebensphasenbezogen die Synchronisierung zwischen privaten und betrieblichen Belangen.

Das Modell „Haus der Arbeitsfähigkeit“ ist flexibel genug, um auch Paketlösungen in dem Sinne möglich zu machen, dass eher von der Arbeitnehmerseite gewünschte gesundheitsbezogene, Belastungen reduzierende und kompensierende Maßnahmen im Paket mit zum Teil eher seitens der Arbeitgeber*innen favorisierten Weiterbildungsanstrengungen auch noch im reiferen Alter vereinbart werden können.

Neben Vorbildern und guten Praxisbeispielen auf höchster Stufe des Personalmanagements (siehe Schaubild „Reifegrad“, Phase 4) ist es von besonderer Wichtigkeit, in bis dato verschleißintensiven und wenig tarifvertraglich geregelten Branchen mit externer fachlicher Hilfe wenigstens die ersten beiden Stufen zu erreichen. Anderenfalls droht eine Situation, in der Arbeitnehmervertretungen im öffentlichen Diskurs eher auf die Probleme der Holzklasse und Arbeitgebervertretungen auf die Errungenschaften der Champagnerklasse verweisen, und dies das Bild weiterer Segmentierung in abgesicherte Kern- und zwangsflexibilisierte randständige Arbeitsmarktsegmente zementieren würde. Prozesstarifverträge sind einer Schritt-für-Schritt-Entwicklung hin zu einer menschengerechteren Arbeitswelt dienlich, mahnen Lernende Systeme an und verhindern kurzfristige Überforderung.

Wenn die Arbeitsformate hinreichend menschengerecht sind, ließe sich sogar mit Blick in skandinavische Länder ${ }^{56}$ darüber nachdenken, mit welchen Maßnahmen es gelingen kann, Personen auch noch über ihr offiziel- 
les Rentenalter hinaus auf freiwilliger Basis zum Weiterarbeiten zu motivieren.

Anliegen der Tarifparteien muss es sein, weder durch die Corona-Krise noch durch die Transformation hin zum Arbeiten 4.0 und einer digitalisierten Arbeitswelt Kriterien guter Arbeit infrage stellen zu lassen ${ }^{57}$, und stattdessen immer wieder neu zu diskutieren, was gute Arbeit unter modifizierten Bedingungen ausmacht und wie sich eine hinreichende soziale Absicherung herstellen lässt. Tarifpolitisches wie arbeitswissenschaftliches Know-how hilft wesentlich dabei, die Aushandlungsprozesse zur Gestaltung der Arbeit und ihrer rechtlichen Rahmenbedingungen zu organisieren und zu moderieren, zu kanalisieren und letztlich in Tarifverträgen und Betriebsvereinbarungen zu fixieren, deren Umsetzung zu überwachen und ggf. Nachsteuerungen in die Wege zu leiten. Hierin ist auch der große Nutzen der letztjährigen Entwicklungen zu sehen, denn die Sozialpartner haben im Zuge der veränderten Arbeitswelt das „Handlungsrepertoire“ ausgedehnt und gelernt, strategisch neue Anforderungen an tarifliche Vereinbarungen (Qualifizierung, Demografie, Gesundheit, Langzeitkonten etc.) zu implementieren ${ }^{58}$. Nur wenn das erarbeitete Instrumentarium strategisch zielführend genutzt wird, wird es möglich werden, Kräften, die die Corona-Krise dazu nutzen möchten, Arbeitnehmerrechte zurückzufahren und einem deregulierten Neoliberalismus als vermeintlicher Krisenlösung das Wort reden, konstruktive und faire tarifpartnerschaftliche Lösungsansätze entgegenzusetzen. Derartige Ansätze schaffen Orientierung in schwieriger Zeit, grenzen existenzielle Ängste ein und verhindern dadurch einen Nährboden für extremistische und demokratiegefährdende politische Bestrebungen.

\section{Literatur}

Balodis, H.; Hühne, D. (2017): Die große Rentenlüge: Warum eine gute und bezahlbare Alterssicherung für alle möglich ist. Westend-Verlag Frankfurt a. M.

Bauer, M.; Guggemos, P. (2004): Schwungfeder - Empowerment für die nachberufliche Phase. AIP-Verlag Augsburg.

Behrens, B.; Kühn, M. (2013): Potenziale von Menschen mit Behinderung erkennen, fördern und einsetzen - Das ganzheitliche Personalmanagement der Bundesagentur für Arbeit (Nürnberg). In: S. A. Boehm, M. K. Baumgärtner, D. J. G. Dwertmann (Hrsg.): Berufliche Inklusion von Menschen mit Behinderung: Best Practices aus dem ersten Arbeitsmarkt. V. Springer Gabler Berlin und Heidelberg, S. 169-182. 
Bellmann, L.; Brandl, S.; Dummert, S.; Guggemos, P.; Leber, U.; Matuschek, I. (2018): Altern in Betrieb. (EBA-Abschlussbericht). Hans Böckler Stiftung (Reihe Forschungsförderung Nr. 393, August 2018), unter: https://www.boeckler.de/pd f/p_study_hbs_393.pdf (Zugriff 11.6.2020).

Birchmeier, U. (2004): DP Diskussionspapier. Die Zweitkarriere nach 50. Möglichkeiten, Wege und Perspektiven. $<$ Schweizer $>$ Staatssekretariat für Wirtschaft, Direktion für Wirtschaftspolitik (DP), unter: http://www.gesunde-maenner.ch/dat a/data_266.pdf (Zugriff 11.6.2020).

BMAS, Ausschuss für Arbeitsmedizin (Hrsg.) (2018): Arbeitsmedizin. Erhalt der Beschäftigungsfähigkeit. Arbeitsmedizinische Empfehlung, bearb. von J. Bischof et al. Bonn, unter: http:/www.bmas.de/SharedDocs/Downloads/DE/PDF-Publik ationen-DINA4/a452-erhalt-beschaeftigungsfaehigkeit.pdf?_blob=publicationFil e (Zugriff 11.6.2020).

BMFSFJ; IW Köln (2005): Familienfreundliche Regelungen in Tarifverträgen und Betriebsvereinbarungen. Erstellt von Chr. Flüter-Hoffmann. BMFSFJ Berlin, unter: https:/www.bmfsfj.de/blob/76376/34cf5d066c64a0f498ed612b45a6b1a7/fam ilienfreundliche-regelungen-data.pdf (Zugriff 11.6.2020).

BMFSFJ (2015): Familienbilder in Deutschland und Frankreich. Monitor Familienforschung. Beiträge aus Forschung, Statistik und Familienpolitik. BMFSFJ Berlin.

Brandl, S.; Guggemos, P.; Matuschek, I. (2018): Vom Einzelfall zum systematischen Alter(n)smanagement in KMU. WSI-Mitteilungen 71(1), S. 51-58.

Conrads, R.; Felder, R. (2017): Wandel gestalten - ein Parcours der Arbeitsfähigkeit in der Druckmaschinenindustrie. In: M. Giesert, T. Reuter, A. Liebrich (Hrsg.): Arbeitsfähigkeit 4.0. Eine gute Balance im Dialog gestalten. VSA-Verlag Hamburg, S. 180-195.

Conrads, R.; Giesert, M.; Liebrich, A.; Reuter, T. (2014): Arbeitsfähigkeitsmanagement im Demographischen Wandel. Ein Leitfaden für Unternehmen und Beschäftigte im Demographischen Wandel. Stadtbergen.

Conrads, R.; Guggemos, P.; Klevenow, G.-H. (2020): Gute Arbeit 4.0 - Was könnte "gute Arbeit" unter Bedingungen des Arbeitens 4.0 bedeuten? In: Th. Freiling, R. Conrads, A. Müller-Osten, J. Porath (Hrsg.): Zukünftige Arbeitswelten. Facetten guter Arbeit, beruflicher Qualifizierung und sozialer Sicherung. Springer Fachmedien Wiesbaden, S. 3-39.

Crouch, C. (2019): Gig Economy. Prekäre Arbeit im Zeitalter von Uber, Minijobs \& Co. V. Suhrkamp Berlin.

Debler, Chr.; Leunig, Chr.; Osterwald, J.; Schlegel, U. (2018): Karriere 50plus neue Perspektiven schaffen. Erste Ergebnisse einer qualitativen Branchenerhebung. In: WSI-Mitteilungen 1/2018, S. 59-65.

DIW Berlin (2018): Stupsen und Schubsen (Nudging): Ein neues verhaltensbasiertes Regulierungskonzept? Vierteljahreshefte zur Wirtschaftsforschung 1/87. Jg., V. Duncker \& Humblot Berlin.

EVG Demografietarifvertrag Runde 2018, unter: https://www.evg-online.org/filead min/Tarif/DemografieTV/evg_TR18_TV_DemografieTV_DB_AG_190508.pdf (Zugriff 11.6.2020). 
Foliensatz zum CLARA-Projekt des IFS mit der DB, unter https://www.iga-info.de/ fileadmin/redakteur/Veranstaltungen/iga.Kolloquium/10._iga.Kolloquium/PDFDokumente/10igaKolloquium_Forum-II_Mouget_CLARA.pdf (Zugriff 11.6.2020).

Flughafen München GmbH, Betriebsrat (Hrsg.) (2017): Das „Münchner Modell“ Vorbild für die ganze Branche. Ein Fachreader zum Symposium. Corporate Media München.

Freidank, J.; Grabbe, J.; Kädtler, J.; Tullius, K. (2011): Altersdifferenzierte und alternsgerechte Betriebs- und Tarifpolitik. Eine Bestandsaufnahme betriebspolitischer und tarifvertraglicher Maßnahmen zur Sicherung der Beschäftigungsfähigkeit. Reihe INQA-Berichte.

Fröhler, N.; Fehmel, T. (2018): Tarifvertragliche Regelung sozialer Sicherung. Deutschland und Österreich im Vergleich. HBS Working Paper Forschungsförderung Nr. 76, Juni 2018. Hans Böckler Stiftung Düsseldorf.

Fuchs, T. et al. (2016): Abschlussbericht des Demografie- und Tarifprojekts ZusammenWachsen - ArbeitGestalten (Branchenberichte). Gesellschaft für Gute Arbeit Grassau, unter: https://40663509-b24b-4cde-921a-06ad4dc7c1db.filesusr.com /ugd/7f6e8d_ce15c07e312049e38decfdb9518d36d8.pdf (Zugriff 11.6.2020).

Fuchs, T.; Backmund, M. (o. J.): Arbeitszeitgestaltung - zum Erhalt der Arbeitsund Beschäftigungsfähigkeit. Eine exemplarische Übersicht nationaler und europäischer Betriebs- und Regelungspraxis. Gesellschaft für Gute Arbeit München und Grassau.

Georg, A.; Katenkamp, O.; Martens, H.; Naegele, G. (2011): Evaluation des Tarifvertrages „Demographischer Wandel“ in der Eisen- und Stahlindustrie. Bericht an die Hans Böckler Stiftung. Projekt-Nr. 2008-111-3. Dortmund.

Gerhard, U.; Pommerenke, P.; Wischermann, U. (Hrsg.) (2008): Klassikerinnen feministischer Theorie Band I: Grundlagentexte (1789-1919). Ulrike Helmer Verlag Sulzbach am Taunus.

Hafesbrink, J.; Krause, M. (2017): Age-Management. Den demografischen Wandel in Unternehmen gestalten. Haufe Verlag Freiburg, München und Stuttgart.

Haipeter, T. (2019): Interessenvertretung in der Industrie 4.0. Das gewerkschaftliche Projekt Arbeit 2020. Nomos Verlag Baden-Baden.

Hentze, H.; Seniorenvertretung Münster (2010): Projekt „Ich gehe jetzt - in eine neue Zukunft!“ (2009/10), unter: https://www.fh-muenster.de/wirtschaft/.../hent ze/Endfassung_Bericht.pdf (Zugriff 11.6.2020).

INQA-Geschäftsstelle (Hrsg.) (2014): Monitor Tarifverträge zur Gestaltung der Qualität der Arbeit. Ein aktueller Überblick über Vereinbarungen zu alternsgerechten und demografiefesten Arbeitsbedingungen. Erarbeitet von der Gesellschaft für Gute Arbeit mbH. Berlin.

Katenkamp, O.; Martens, H.; Georg, A. (2012): Gestaltung des demografischen Wandels durch Tarifvertrag? In: WSI-Mitteilungen 6/2012, S. 427-434. 
Kricheldorff, C. (2011): Vom Erwerbsleben ins Engagement - Grundhaltungen in der Statuspassage zur nachberuflichen Phase und deren Verknüpfung mit geragogischen Konzepten und Settings. In: Informationsdienst Altersfragen 38 (5), S. 12-19, unter: https://www.dza.de/fileadmin/dza/pdf/Heft_05_2011_Septembe r_Oktober_2011_gekuerzt_PW.pdf (Zugriff 11.6.2020).

Latniak, E.; Voss-Dahm, D. (2010): Umsetzung demografiefester Personalpolitik in der Chemischen Industrie. Inhaltliche und prozessuale Analyse betrieblicher Vorgehensweisen. Ergebnisse der Begleitforschung zum INQA-Projekt deci. Forschungsprojekt HBS 2008-110-63 (zit. als „deci-Abschlussbericht“).

Lesch, H.; Mayer, A.; Schmid, L. (2014): Demografischer Wandel und Tarifpolitik. Forschungsbericht aus dem IW Nr. 96. Köln.

Lesch, H.; Mayer, A.; Schmid, L. (2019): Arbeitszeitpolitik: Die neuen tarifpolitischen Herausforderungen. In: IW-Trends 2/2019, S. 103-120.

Lottenbach, A.; Perroulaz, E. (2013): SBB anyway solutions (Bern) - Gelungene berufliche Reintegration bei den schweizerischen Bundesbahnen. In: S. A. Boehm, M. K. Baumgärtner, D. J. G. Dwertmann (Hrsg.): Berufliche Inklusion von Menschen mit Behinderung: Best Practices aus dem ersten Arbeitsmarkt. V. Springer Gabler Berlin und Heidelberg, S. 183-194.

Mangelsdorf, M. (2017): Von Babyboomer bis Generation Z. Der richtige Umgang mit unterschiedlichen Generationen im Unternehmen. V. GABAL Offenbach.

Mouget, S.; Wolff, S. (2016): Berufliche Leistungsfähigkeit im Alter erhalten und aktiv den Übergang in den Ruhestand gestalten - Ansätze im DB-Konzern. In: Berufsverband Deutscher Psychologinnen und Psychologen e. V. (Hrsg.): Psychologie - Gesellschaft - Politik 2016. Älter werden - gesund bleiben. BDP Bundesgeschäftsstelle Berlin, S. 33-37.

Münzel, T.; Neuhaus, T. (2013): Das Integrationsmanagement der AUDI AG (Ingolstadt). In: S. A. Boehm, M. K. Baumgärtner, D. J. G. Dwertmann (Hrsg.): Berufliche Inklusion von Menschen mit Behinderung. Best Practices aus dem ersten Arbeitsmarkt. V. Springer Gabler Berlin und Heidelberg, S. 113-120.

Nahles, A. (2016): Arbeiten 4.0 - Perspektiven einer neuen Arbeitszeitpolitik. In: G. Bäcker, S. Lehndorff, C. Weinkopf (Hrsg.): Den Arbeitsmarkt verstehen, um ihn zu gestalten. Festschrift für Gerhard Bosch. Springer Vs. Wiesbaden, S. 37-47.

Neue Caritas (2015): Familienpolitik lässt sich nicht exportieren. Wie lassen sich Armut und soziale Ausgrenzung von Kindern, Alleinerziehenden und benachteiligten Familien vermeiden? Ein Vergleich der Familienpolitik in Deutschland, Dänemark, Frankreich und Slowenien gibt Anhaltspunkte für Lösungsmöglichkeiten, unter: https:/www.caritas.de/neue-caritas/heftarchiv/jahrgang20 14/artikel/familienpolitik-laesst-sich-nicht-exportieren (Zugriff 11.6.2020).

Projektgruppe INDIGHO (2013): Demografischer Wandel im Gastgewerbe: Kompetenzentwicklung und Qualifizierung unter Bedingungen diskontinuierlicher Beschäftigung. Arbeitspapier 1. TU Dortmund, Sozialforschungsstelle, Band 187. 
Rauchecker, J. (2016): Baby Boomer, Generation X und Generation Y. Gibt es Unterschiede in den Arbeitswerten, Arbeitseinstellungen und Arbeitsverhaltensweisen dieser drei Generationen? Akademikerverlag Saarbrücken.

Reuyß, S., Rauschnick, L. \& Kanamüller, A. (2016): Arbeitszeit. Qualitative Ergebnisse für Deutschland. Expertise für die Kommission „Arbeit der Zukunft“. Hans Böckler Stiftung.

Russell Hochschild, A.; Ehrenreich, B. (2004): Global Woman: Nannies, Maids, and Sex Workers in the New Economy. Henry Holt New York.

Schmierl, K.; Weimer, S. (2014): Demografiesensible Entgeltpolitik. Annäherungen an ein Zukunftsthema. Springer Vs. Wiesbaden.

Stuber, M. (2004): Diversity. Das Potenzial von Vielfalt nutzen - den Erfolg durch Offenheit steigern. V. Luchterhand/Wolters Kluwer München.

Tempel, J.; Ilmarinen, J. (Hrsg.) (2013): Arbeitsleben 2025. Das Haus der Arbeitsfähigkeit im Unternehmen bauen. VSA Hamburg.

Themenheft WSI-Mitteilungen 1/2018, hrsg. von L. Bellmann, S. Brandl u. I. Matuschek.

Tullius, K.; Freidank, J.; Grabbe, J.; Kädtler, J.; Schroeder, W. (2012): Perspektiven alter(n)sgerechter Betriebs- und Tarifpolitik. In: WSI-Mitteilungen 2/2012, S. 113-123.

Ulich, E. (2016): Differenzielle Arbeitsgestaltung - ein zukunftsfähiges Konzept. In: Institut für Arbeitsforschung und Organisationsberatung (Hrsg.): Unternehmensgestaltung im Spannungsfeld von Stabilität und Wandel. vdf Hochschulverlag AG an der ETH Zürich, Zürich, S. 159-175.

Unruh, T. (Hrsg.) (1989): Tatort Pflegeheim. Zivildienstleistende berichten. Klartextverlag Essen

ver.di, Bereich Innovation und gute Arbeit (Hrsg.) (2019): Arbeitsintensität - Perspektiven, Einschätzungen, Positionen aus gewerkschaftlicher Sicht. Innovation und gute Arbeit. ver.di Berlin.

WSI-Tarifarchiv (verschiedene Jahrgänge).

ZusammenWachsen-ArbeitGestalten - das Demografie- und Tarifprojekt, unter: www.zusammenwachsen-arbeitgestalten.de (Zugriff 11.6.2020).

\section{Referenzen}

1 Wir sprechen in diesem Aufsatz von „Demografiemanagement“, und thematisieren damit auch Vereinbarkeitsfragen, Bedürfnisse von Menschen mit Migrationsoder Fluchthintergrund. Eine Zusammenfassung der Diskussion zum Defizitund Kompetenzmodell des Alters geben Freidank et al. 2011: Altersdifferenzierte und alternsgerechte Betriebs- und Tarifpolitik. Eine Bestandsaufnahme betriebspolitischer und tarifvertraglicher Maßnahmen zur Sicherung der Beschäftigungsfähigkeit. Reihe INQA-Berichte 42. Dortmund u. Berlin, S. $24 \mathrm{f}$.

2 So könnten beispielsweise Vertretungen aus dem Management die Perspektive, „länger arbeiten (müssen)“, betonen, und Beschäftigte dennoch auf vorzeitigen 
Altersübergang orientiert bleiben. Es geht nicht nur um die Ausstiegsoption, sondern auch um die Freiheit, ggf. vorher gehen zu können, so einem Veränderungsprozesse, Vorgesetztenverhalten o. Ä. nicht mehr gefallen würden. Vorruhestandsmöglichkeiten kommt damit auch die Rolle eines Flexibilitätspuffers und der Vermittlung eines gewissen Freiheits- und Unabhängigkeitsgefühls im Kopf zu. Es macht dabei einen enormen Unterschied, ob jemand weiterarbeiten „muss" oder „kann/darf“. Zur nachwirkenden Orientierung auf Frühverrentung vgl. u. a. Erich Latniak \& Dorothea Voss-Dahm 2010: Umsetzung demografiefester Personalpolitik in der Chemischen Industrie. Inhaltliche und prozessuale Analyse betrieblicher Vorgehensweisen. Ergebnisse der Begleitforschung zum INQA-Projekt deci. Forschungsprojekt HBS 2008-110-63, S. 13. Ein weiteres Beispiel für einen Deutungsrahmen könnte sein, dass bestehende personalpolitische Instrumente im eigenen Betrieb ausreichen, um neuen Herausforderungen wie älteren und bunteren Belegschaften zu genügen. Denkbar wäre auch die Überzeugung, dass Ältere ja wertgeschätzt werden - ohne dass deshalb in deren Humanressourcen wie Qualifizierung und Gesundheit besonders investiert werden müsste.

3 Befunde des qualitativen Teils der EBA-Studie. Bellmann et al. 2018: Altern in Betrieb. (EBA-Abschlussbericht). Hans Böckler Stiftung. (Reihe Forschungsförderung Nr. 393, August 2018), Download unter: https://www.boeckler.de/pdf/p_stu dy_hbs_393.pdf.

4 So können z.B. bestimmte Beschäftigungs- oder auch hohe Besoldungsgruppen von manchen Regelungen ausgenommen sein.

5 Vgl. N. Fröhler \& T. Fehmel 2018: Tarifvertragliche Regelung sozialer Sicherung. Deutschland und Österreich im Vergleich. HBS Working Paper Forschungsförderung Nr. 76, Juni 2018. Hans Böckler Stiftung Düsseldorf.

6 Vgl. N. Fröhler \& T. Fehmel 2018: Tarifvertragliche Regelung sozialer Sicherung. Deutschland und Österreich im Vergleich. HBS Working Paper Forschungsförderung Nr. 76, Juni 2018. Hans Böckler Stiftung Düsseldorf, S. 27-46. Siehe zum deutsch-österreichischen Rentenvergleich auch: H. Balodis \& D. Hühne 2017: Die große Rentenlüge: Warum eine gute und bezahlbare Alterssicherung für alle möglich ist. Westend-Verlag Frankfurt a. M.; Balodis \& Hühne loben das rein staatliche österreichische Rentenversicherungssystem, kritisieren den deutschen Einstieg in kapitalgedeckte Absicherungsformen und fordern hieraus wieder auszusteigen.

7 Bellmann et al. 2018: Altern in Betrieb. (EBA-Abschlussbericht). Hans Böckler Stiftung. (Reihe Forschungsförderung Nr.393, August 2018), Download unter: https://www.boeckler.de/pdf/p_study_hbs_393.pdf.

8 Vgl. hierzu neben den grundsätzlichen Optionen und Handlungsmöglichkeiten, dargestellt in: INQA-Geschäftsstelle 2014: Monitor Tarifverträge zur Gestaltung der Qualität der Arbeit. Ein aktueller Überblick über Vereinbarungen zu alternsgerechten und demografiefesten Arbeitsbedingungen. Berlin, S. 13, die Maßnahmen der Schweizer Bundesbahnen für Personen mit bereits manifestierter Leistungswandlung: A. Lottenbach \& E. Perroulaz 2013: SBB anyway solutions (Bern) - Gelungene berufliche Reintegration bei den schweizerischen Bundesbahnen, in: S. A. Boehm, M. K. Baumgärtner \& D. J. G. Dwertmann (Hrsg.): Berufliche Inklusion von Menschen mit Behinderung: Best Practices aus dem ersten Arbeitsmarkt. V. Springer Gabler Berlin und Heidelberg, S. 183-194. 
9 Vgl. hierzu K. Schmierl \& S. Weimer 2014: Demografiesensible Entgeltpolitik. Annäherungen an ein Zukunftsthema. Springer VS Wiesbaden.

10 Vgl. ver.di, Bereich Innovation und gute Arbeit (Hrsg.) 2019: Arbeitsintensität Perspektiven, Einschätzungen, Positionen aus gewerkschaftlicher Sicht. Innovation und gute Arbeit. ver.di Berlin.

11 S. Skrabs 2019: Arbeitsintensität aus tarifpolitischer Perspektive, in: ver.di 2019: Arbeitsintensität - Perspektiven, Einschätzungen, Positionen aus gewerkschaftlicher Perspektive. ver.di Berlin, S. 160-165.

12 Klassische Studien zur Emotionsarbeit von A. R. Hochschild (zus. mit B. Ehrenreich 2004: Global Woman: Nannies, Maids, and Sex Workers in the New Economy. Henry Holt New York).

13 Vgl. Zitat von P. Hausmann von der IGBCE in: INQA-Geschäftsstelle 2014: Monitor Tarifverträge zur Gestaltung der Qualität der Arbeit. Ein aktueller Überblick über Vereinbarungen zu alternsgerechten und demografiefesten Arbeitsbedingungen. Berlin, S. 15.

14 BMFSFJ 2015: Familienbilder in Deutschland und Frankreich. Monitor Familienforschung. Beiträge aus Forschung, Statistik und Familienpolitik. BMFSFJ Berlin, S. 18 (nach Vergleichszahlen von Eurostat 2014). Französische Frauen arbeiten mit einem Kind in relativ ähnlichem Umfang wie kinderlose Frauen, und insgesamt weit seltener in Teilzeit als in Deutschland.

15 Vgl. u.a. A. Nahles 2016: Arbeiten 4.0 - Perspektiven einer neuen Arbeitszeitpolitik, in: G. Bäcker, S. Lehndorff \& C. Weinkopf (Hrsg.): Den Arbeitsmarkt verstehen, um ihn zu gestalten. Festschrift für Gerhard Bosch. Springer Vs. Wiesbaden, S. 37-47. Nahles mahnt mehr Flexibilisierungsmöglichkeiten für Arbeitgeber*innen und Arbeitnehmer*innen an, auch mit Hochfahren der Arbeitszeiten der Frauen und Reduzieren der Arbeitsvolumina der Männer, die an die Stelle einer weiteren Arbeitszeitverkürzung für alle treten sollten.

16 Dies zeigt sich im Vergleich zu Dänemark und Frankreich, wo sowohl die Familienpausen kurz wie eine frühere Rückkehr in eine Vollzeittätigkeit üblich sind. Vgl. Kurzdarstellung: Neue Caritas 2015: Familienpolitik lässt sich nicht exportieren. Wie lassen sich Armut und soziale Ausgrenzung von Kindern, Alleinerziehenden und benachteiligten Familien vermeiden? Ein Vergleich der Familienpolitik in Deutschland, Dänemark, Frankreich und Slowenien gibt Anhaltspunkte für Lösungsmöglichkeiten. Download unter: https://www.caritas.de/neue-caritas/ heftarchiv/jahrgang2014/artikel/familienpolitik-laesst-sich-nicht-exportieren.

17 Vgl. die Befunde der KarisMa-Studie von Chr. Debler et al. 2018, S. 63 f. sowie den Monitor Tarifverträge zur Gestaltung der Qualität der Arbeit der INQA-Geschäftsstelle 2014.

18 Bellmann et al. 2018: Altern in Betrieb. (EBA-Abschlussbericht). Hans Böckler Stiftung. (Reihe Forschungsförderung Nr.393, August 2018), Download unter: https://www.boeckler.de/pdf/p_study_hbs_393.pdf.

19 U. a. empfehlen Chr. Debler et al. 2018: Karriere 50plus - neue Perspektiven schaffen. Erste Ergebnisse einer qualitativen Branchenerhebung, in: WSI-Mitteilungen 1/2018, S. 59-65 „Karriereberatungen und Bilanzworkshops“. (S. 62).

20 Projektgruppe INDIGHO 2013: Demografischer Wandel im Gastgewerbe: Kompetenzentwicklung und Qualifizierung unter Bedingungen diskontinuierlicher Beschäftigung. Arbeitspapier 1. TU Dortmund, Sozialforschungsstelle, Band 187.

21 Vgl. zu Weiterbildungshürden bei Älteren M. Morschhäuser 2006: Reife Leistung: Personal- und Qualifizierungspolitik für die künftige Altersstruktur. Editi- 
on Sigma Berlin; zu digitalem Stress und Weiterbildungsdruck für Ältere vgl. ver.di 2019: Arbeitsintensität - Perspektiven, Einschätzungen, Positionen aus gewerkschaftlicher Sicht. Innovation und gute Arbeit. ver.di Berlin. Auf mehrere Studienbefunde zu deutlich geringeren Bestrebungen zur Weiterbildung Älterer verweist der deci-Abschlussbericht 2010 von Latniak \& Voss-Dahm 2010, S. 15.

22 So konnten Jüngere beispielsweise gleichviele Urlaubstage wie Ältere einklagen. Hier zeigt sich auch das bereits angesprochene Implementationsproblem vorliegender Konzeptionen. Denn im Sinne einer individuellen Balance von Anforderungen und individuellen Eigenschaften, wie es beispielsweise das Modell der Arbeitsbewältigungsfähigkeit nach Ilmarinen und Tempel (2013) vorsieht, würde es um eine Gleichbehandlung in vergleichbaren Arbeitsfähigkeitskonstellationen gehen. Das geht weit über den Vergleich von Alterskohorten hinaus.

23 Bellmann et al. 2018: Altern in Betrieb. (EBA-Abschlussbericht). Hans Böckler Stiftung. (Reihe Forschungsförderung Nr.393, August 2018), Download unter: https://www.boeckler.de/pdf/p_study_hbs_393.pdf.

24 H. Hentze \& Seniorenvertretung Münster 2010: Projekt „Ich gehe jetzt - in eine neue Zukunft!“ (2009/10), im Internet unter: https://www.fh-muenster.de/wirtsc haft/.../hentze/Endfassung_Bericht.pdf.

$25 \mathrm{Zu}$ ähnlichen Interview-Befunden kam die KarisMa-Studie (aus der ChemieBranche), die für Entwicklungsmöglichkeiten ab 50 plädiert, ohne dass diese mit klassisch-hierarchischen Aufstiegskarrieren identisch sein müssten, diese aber auch nicht ausschließen sollten. Wichtig ist dabei auch der Hinweis auf u. U. spätere berufliche Entwicklungen von Frauen nach karrieretechnischer Zurückhaltung während flankierender Familienpflichten. Vgl. Chr. Debler et al. 2018: Karriere 50plus - neue Perspektiven schaffen. Erste Ergebnisse einer qualitativen Branchenerhebung, in: WSI-Mitteilungen 1/2018, S. 59-65.

26 Stecker et al. 2011: GeniAL - Ein neuer Arbeitgeberservice der Deutschen Rentenversicherung zur Unterstützung für ein demografiefestes Unternehmen. In: RV aktuell, 7/2011, S. 197-202.

27 R. Conrads \& R. Felder 2017: Wandel gestalten - ein Parcours der Arbeitsfähigkeit in der Druckmaschinenindustrie. In: M. Giesert, T. Reuter, \& A. Liebrich (Hrsg.): Arbeitsfähigkeit 4.0. Eine gute Balance im Dialog gestalten. VSA-Verlag Hamburg, S. 180-195.

28 Vgl. U. Gerhard, P. Pommerenke \& U. Wischermann (Hrsg.) 2008: Klassikerinnen feministischer Theorie Band I: Grundlagentexte (1789-1919) Ulrike Helmer Verlag Sulzbach am Taunus.

29 Vgl. hierzu den Beitrag von W. Anlauft et al. in diesem Band.

30 Einen wichtigen Beitrag zur Weiterentwicklung von tarifvertraglichen Lösungen im demografischen Wandel in Dienstleistungsbranchen leistete das Demografieund Tarifprojekt ZusammenWachsen-ArbeitGestalten. Zu den tarifvertraglichen Regelungen siehe T. Fuchs \& M. Backmund (o.J.): Arbeitszeitgestaltung - zum Erhalt der Arbeits- und Beschäftigungsfähigkeit. Eine exemplarische Übersicht nationaler und europäischer Betriebs- und Regelungspraxis. Gesellschaft für Gute Arbeit, München u. Grassau, S. 13 zum Tarifvertrag der Verkehrsbetriebe Hamburg-Holstein.

$31 \mathrm{Zu}$ den tarifvertraglichen Regelungen siehe T. Fuchs \& M. Backmund (o.J.): Arbeitszeitgestaltung - zum Erhalt der Arbeits- und Beschäftigungsfähigkeit. Eine exemplarische Übersicht nationaler und europäischer Betriebs- und Regelungspraxis. Gesellschaft für Gute Arbeit, München u. Grassau, S. 9. zum Tarifvertrag 
zu Langzeitkonten und Demografie für die Arbeitnehmerinnen der Universitätsklinika Freiburg, Heidelberg, Tübingen und Ulm. Zur Deutung der Chronologie von Demografieverträgen siehe N. Fröhler \& T. Fehmel, 2018: Tarifvertragliche Regelung sozialer Sicherung. Deutschland und Österreich im Vergleich. HBS Working Paper Forschungsförderung Nr.76, Juni 2018. Hans Böckler Stiftung Düsseldorf, S. 20 - 26.

32 So wurden 2016 Gefährdungsbeurteilungen bei IBM tarifvertraglich geregelt, u.a. mit einer Gesundheitskommission und der Rückverlagerung der Verantwortung für die Einhaltung der Arbeitszeit an Führungskräfte. Vgl. ver.di 2019, S. 163.

33 Beispiel Rhein-Neckar-Verkehr seit 10/2016: Ab 55 Jahren 6,5 Tage Freistellung, bis hin zu 51 Tagen mit 64 Jahren, bei 97\% Lohnkompensation, plus eines freiwilligen Zeitwertkontos. (Siehe ver.di 2019, S. 163 f.).

34 ver.di 2019: Arbeitsintensität - Perspektiven, Einschätzungen, Positionen aus gewerkschaftlicher Sicht. Innovation und gute Arbeit. ver.di Berlin, S. $163 \mathrm{f}$.

35 ver.di 2019: Arbeitsintensität - Perspektiven, Einschätzungen, Positionen aus gewerkschaftlicher Sicht. Innovation und gute Arbeit. ver.di Berlin, S. $164 \mathrm{f}$.

36 Vgl. K. Schmierl \& S. Weimer 2014: Demografiesensible Entgeltpolitik. Annäherungen an ein Zukunftsthema. Springer VS Wiesbaden.

37 Aus heutiger Sicht interessant sind frühe Regelungen in Tarifverträgen zur Familienfreundlichkeit, die teilweise bis in die 1990er-Jahre zurückgehen, in: BMFSFJ \& IW Köln 2005: Familienfreundliche Regelungen in Tarifverträgen und Betriebsvereinbarungen. Erstellt von Chr. Flüter-Hoffmann. BMFSFJ Berlin, Download unter: https://www.bmfsfj.de/blob/76376/34cf5d066c64a0f498ed612b45a6b1 a7/familienfreundliche-regelungen-data.pdf.

38 T. Münzel \& T. Neuhaus 2013: Das Integrationsmanagement der AUDI AG (Ingolstadt), in: S. A. Boehm, M. K. Baumgärtner, D. J. G. Dwertmann (Hrsg.): Berufliche Inklusion von Menschen mit Behinderung. Best Practices aus dem ersten Arbeitsmarkt. V. Springer Gabler Berlin und Heidelberg, S. 113-120. Würden Leistungsgewandelte im Gruppenakkord voll gerechnet, könne dies dazu führen, dass Kollegen sie loswerden möchten. Über eine Abbildung der realistischen Leistungsfähigkeit mit einem bestimmten Faktor kleiner 1 im Gruppenakkord lässt sich eine Benachteiligung der anderen Gruppenmitglieder ausschließen. Alleine auf informelle solidarische Lösungen in altersgemischten Teams zu setzen, die es durchaus gibt (Vgl. K. Schmierl \& S. Weimer, Stefanie 2014: Demografiesensible Entgeltpolitik. Annäherungen an ein Zukunftsthema. Springer VS Wiesbaden), reicht bei insgesamt älter werdenden Belegschaften in alternskritischen Tätigkeitsfeldern nicht aus.

39 Vgl. Bellmann et al. 2018: Altern in Betrieb. (EBA-Abschlussbericht). Hans Böckler Stiftung. (Reihe Forschungsförderung Nr. 393, August 2018), Download unter: https://www.boeckler.de/pdf/p_study_hbs_393.pdf.

40 Vgl. z.B. T. Unruh (Hrsg.) 1989: Tatort Pflegeheim. Zivildienstleistende berichten. Klartextverlag Essen.

41 B. Behrens \& M. Kühn 2013: Potenziale von Menschen mit Behinderung erkennen, fördern und einsetzen - Das ganzheitliche Personalmanagement der Bundesagentur für Arbeit (Nürnberg), in: S. A. Boehm, M. K. Baumgärtner \& D. J. G. Dwertmann (Hrsg.) 2013: Berufliche Inklusion von Menschen mit Behinderung: Best Practices aus dem ersten Arbeitsmarkt. V. Springer Gabler Berlin und Heidelberg, S. 169-182. 
42 Vgl. u.a. INQA-Geschäftsstelle (Hrsg.) 2014: Monitor Tarifverträge zur Gestaltung der Qualität der Arbeit. Ein aktueller Überblick über Vereinbarungen zu alternsgerechten und demografiefesten Arbeitsbedingungen. Erarbeitet von der Gesellschaft für Gute Arbeit mbH. Berlin.

43 Vgl. S. Mouget \& S. Wolff 2016: Berufliche Leistungsfähigkeit im Alter erhalten und aktiv den Übergang in den Ruhestand gestalten - Ansätze im DB-Konzern, in: Berufsverband deutscher Psychologinnen und Psychologen e.V. (Hrsg.): Psychologie - Gesellschaft - Politik 2016. Älter werden - gesund bleiben. BDP Bundesgeschäftsstelle Berlin, S. 33-37.

44 Siehe hierzu die Broschüre: Flughafen München GmbH, Betriebsrat (Hrsg.) 2017: Das „Münchner Modell“ - Vorbild für die ganze Branche. Ein Fachreader zum Symposium. Corporate Media München. An diesem Beispiel wird deutlich, was mit arbeitswissenschaftlicher (hier: T. Fuchs und M. Backmund von der Gesellschaft für Gute Arbeit, GFGA) und arbeitsrechtlicher Unterstützung möglich werden kann.

45 Flughafen München GmbH, Betriebsrat 2017: Das „Münchner Modell“ - Vorbild für die ganze Branche. Ein Fachreader zum Symposium. Corporate Media München.

46 R. Conrads \& R. Felder 2017: Wandel gestalten - ein Parcours der Arbeitsfähigkeit in der Druckmaschinenindustrie. In: M. Giesert, T. Reuter \& A. Liebrich (Hrsg.): Arbeitsfähigkeit 4.0. Eine gute Balance im Dialog gestalten. VSA-Verlag Hamburg, S. 180-195.

47 Projektgruppe INDIGHO 2013: Demografischer Wandel im Gastgewerbe: Kompetenzentwicklung und Qualifizierung unter Bedingungen diskontinuierlicher Beschäftigung. Arbeitspapier 1. TU Dortmund, Sozialforschungsstelle, Band 187.

48 Vgl. E. Latniak \& D. Voss-Dahm 2010: Umsetzung demografiefester Personalpolitik in der Chemischen Industrie. Inhaltliche und prozessuale Analyse betrieblicher Vorgehensweisen. Ergebnisse der Begleitforschung zum INQA-Projekt deci. Forschungsprojekt HBS 2008-110-63, S. 11, 31, 37.

49 Befund bereits im deci-Abschlussbericht. Vgl. Latniak \& Voss-Dahm 2010, S. $11 \mathrm{f} ., 15,18,49$.

50 Bellmann et al. 2018: Altern in Betrieb. (EBA-Abschlussbericht). Hans Böckler Stiftung. (Reihe Forschungsförderung Nr.393, August 2018), Download unter: https://www.boeckler.de/pdf/p_study_hbs_393.pdf; Brandl et al. 2018; Brandl et al. 2018: Vom Einzelfall zum systematischen Alter(n)smanagement in KMU. WSI-Mitteilungen 71(1): 51-58.

51 Latniak \& Voss-Dahm 2010 sprechen von einem „Meilenstein innovativer Tarifpolitik“" (S. 51). Besonders sind hier die überbetriebliche Regulierung von Fonds mit unterschiedlicher Nutzungsmöglichkeit, das Vorsehen von Auffanglösungen und der perspektivische Blick auf Unternehmen gemeint, die sich mit externer Hilfe zu Lernenden Organisationen entwickeln können. Ähnlich äußern sich Fröhler \& Fehmel, 2018: Tarifvertragliche Regelung sozialer Sicherung. Deutschland und Österreich im Vergleich. HBS Working Paper Forschungsförderung Nr. 76, Juni 2018. Hans Böckler Stiftung Düsseldorf, S. 20-26.

52 H. Hentze \& Seniorenvertretung Münster 2010: Projekt „Ich gehe jetzt - in eine neue Zukunft!“ (2009/10), im Internet unter: https://www.fh-muenster.de/wirtsc haft/.../hentze/Endfassung_Bericht.pdf.

53 K. Schmierl \& S. Weimer 2014: Demografiesensible Entgeltpolitik. Annäherung an ein Zukunftsthema. Springer Vs. Wiesbaden, S. 159. 
54 Brandl et al. 2018: Vom Einzelfall zum systematischen Alter(n)smanagement in KMU. WSI-Mitteilungen 71(1): 51-58.

55 Vgl. z.B.: M. Mangelsdorf 2017: Von Babyboomer bis Generation Z. Der richtige Umgang mit unterschiedlichen Generationen im Unternehmen. V. GABAL Offenbach; Jessica Rauchecker 2016: Baby Boomer, Generation X und Generation Y. Gibt es Unterschiede in den Arbeitswerten, Arbeitseinstellungen und Arbeitsverhaltensweisen dieser drei Generationen? Akademikerverlag Saarbrücken.

56 In Schweden ist es innerhalb eines flexiblen Ausstiegskorridors gesetzlich geregelt, dass Unternehmen Beschäftigte, die über 65 hinaus weiterarbeiten möchten, bis zu zwei Jahre lang weiterbeschäftigen müssen. Weiterarbeiten wirkt sich positiv auf die Rentenhöhe aus.

57 Vgl. zum Thema guter Arbeit unter Digitalisierungsbedingungen Conrads et al. 2020.

58 Vgl. T. Haipeter (2019): Interessenvertretung in der Industrie 4.0. Das gewerkschaftliche Projekt Arbeit 2020. Nomos Verlag Baden-Baden, S. 35f. 